\title{
MODIFIED PROJECTION AND THE ITERATED MODIFIED PROJECTION METHODS FOR NONLINEAR INTEGRAL EQUATIONS
}

\author{
LAURENCE GRAMMONT, REKHA P. KULKARNI AND PAULO B. VASCONCELOS
}

Communicated by Kendall Atkinson

\begin{abstract}
Consider a nonlinear operator equation $x-$ $K(x)=f$, where $K$ is a Urysohn integral operator with a smooth kernel. Using the orthogonal projection onto a space of discontinuous piecewise polynomials of degree $\leq r$, previous authors have established an order $r+1$ convergence for the Galerkin solution and $2 r+2$ for the iterated Galerkin solution. Equivalent results have also been established for the interpolatory projection at Gauss points. In this paper, a modified projection method is shown to have convergence of order $3 r+3$ and one step of iteration is shown to improve the order of convergence to $4 r+4$. The size of the system of equations that must be solved, in implementing this method, remains the same as for the Galerkin method.
\end{abstract}

1. Introduction. Let $X$ be a complex Banach space and $K$ a nonlinear compact operator defined on a non empty open subset $O$ of $X$. For $f \in X$, we are interested in a solution of

$$
x-K(x)=f .
$$

We assume that the above equation has a unique solution $\varphi$ in $O$.

Let $X_{n}$ be a sequence of finite-dimensional approximating subspaces of $X$, and let $\pi_{n}$ be a sequence of projections from $X$ to $X_{n}$. If $X$ is a Hilbert space and $\pi_{n}$ is the orthogonal projection from $X$ to $X_{n}$, then in the classical Galerkin method, (1.1) is approximated by

$$
\varphi_{n}^{G}-\pi_{n} K\left(\varphi_{n}^{G}\right)=\pi_{n} f .
$$

2010 AMS Mathematics subject classification. Primary 45L10, 65J15, 65R20.

Keywords and phrases. Urysohn integral operator, Galerkin method, collocation at Gauss points.

The work of the first two authors was partially supported by CEFIPRA, Project No. 4101-1. 2013.

Received by the editors on October 15, 2012, and in revised form on April 21, 
If $\pi_{n}$ is an interpolatory projection, then the collocation solution $\varphi_{n}^{C}$ is obtained by solving

$$
\varphi_{n}^{C}-\pi_{n} K\left(\varphi_{n}^{C}\right)=\pi_{n} f
$$

The above projection methods have been studied extensively in the research literature. See Krasnoselsii [9], Krasnoselskii, et al. [10] and Krasnoselskii and Zabreiko [11].

The iterated Galerkin solution is defined by

$$
\varphi_{n}^{S}=K\left(\varphi_{n}^{G}\right)+f
$$

and the iterated collocation solution is defined in a similar fashion. The iterated projection methods are analyzed in [4].

In $[\mathbf{7}, \mathbf{8}]$, the following modified projection method is proposed:

$$
\varphi_{n}^{M}-K_{n}^{M}\left(\varphi_{n}^{M}\right)=f
$$

where

$$
K_{n}^{M}(x)=\pi_{n} K(x)+K\left(\pi_{n} x\right)-\pi_{n} K\left(\pi_{n} x\right), \quad x \in O .
$$

It is a generalization of the modified projection method in the linear case, which was proposed in [12].

As in the case of the iterated Galerkin method, we perform one step of iteration and define the iterated modified projection solution as

$$
\widetilde{\varphi}_{n}^{M}=K\left(\varphi_{n}^{M}\right)+f
$$

In this paper we consider $K$ to be a Urysohn integral operator. For $r \geq 0$, let $X_{n}$ be a space of piecewise polynomials of degree $\leq r$ with respect to a quasi-uniform partition of $[0,1]$. Let $h$ denote the length of the largest subinterval of the partition. The projection $\pi_{n}$ with range $X_{n}$ is chosen either to be the orthogonal projection or an interpolatory projection, defined on an appropriate space. If the kernel of the Urysohn integral operator $K$ is sufficiently smooth, then in the case of orthogonal projection as well as in the case of the interpolatory projection at the Gauss points, we show that

$$
\left\|\varphi_{n}^{M}-\varphi\right\|_{\infty}=O\left(h^{3 r+3}\right)
$$


and

$$
\left\|\widetilde{\varphi}_{n}^{M}-\varphi\right\|_{\infty}=O\left(h^{4 r+4}\right) .
$$

These estimates are to be compared with the following orders of convergence proved in [4]:

$$
\begin{aligned}
& \left\|\varphi_{n}^{G}-\varphi\right\|_{\infty}=O\left(h^{r+1}\right), \quad\left\|\varphi_{n}^{C}-\varphi\right\|_{\infty}=O\left(h^{r+1}\right), \\
& \left\|\varphi_{n}^{S}-\varphi\right\|_{\infty}=O\left(h^{2 r+2}\right) .
\end{aligned}
$$

They have, in fact, obtained the error estimates for the iterated projection methods in a more general setting of a Urysohn integral operator with Green's function type kernel.

As in the case of linear operators, the size of the system of equations that needs to be solved in order to compute $\varphi_{n}^{M}$ remains the same as in the case of the projection method.

In the case of an interpolatory projection with collocation points which are not Gauss points, we show that

$$
\left\|\varphi_{n}^{M}-\varphi\right\|_{\infty}=O\left(h^{2 r+2}\right) .
$$

The corresponding orders of convergence in the collocation as well as the iterated collocation methods are $r+1$.

For $\delta_{0}>0$, let

$$
\mathcal{B}\left(\varphi, \delta_{0}\right)=\left\{\psi \in X:\|\varphi-\psi\|_{\infty}<\delta_{0}\right\}
$$

For future reference, we describe below a result from Grammont [7].

Let $Y$ be a closed subspace of the Banach space $X$, and let the range of $K$ be contained in $Y$. Let $K$ be Fréchet differentiable on $O$ and the Fréchet derivative of $K$, denoted by $K^{\prime}$, Lipschitz continuous. Let $\pi_{n}$ be a sequence of projections such that $\pi_{n} y \rightarrow y$ as $n \rightarrow \infty, y \in Y$.

Theorem $1.1[7]$. Suppose that $\varphi \in O$ is the unique solution of (1.1) with $f=0$ and that 1 is not an eigenvalue of $K^{\prime}(\varphi)$. Then there exists a neighborhood $\mathcal{B}\left(\varphi, \delta_{0}\right)$ of $\varphi$ which contains, for all $n$ large enough, a unique solution $\varphi_{n}^{M}$ of (1.2). In addition,

$$
\frac{2}{3} \alpha_{n} \leq\left\|\varphi_{n}^{M}-\varphi\right\|_{\infty} \leq 2 \alpha_{n}
$$


where $\alpha_{n}=\left\|\left(I-\left(K_{n}^{M}\right)^{\prime}(\varphi)\right)^{-1}\left(K(\varphi)-K_{n}^{M}(\varphi)\right)\right\|$ is a sequence converging to zero. Also,

$$
\frac{\alpha_{n}}{\left\|\pi_{n} \varphi-\varphi\right\|_{\infty}} \longrightarrow 0 \quad \text { as } n \rightarrow \infty
$$

Note that $\varphi_{n}^{M}$ exhibits superconvergence, which is not always the case in the iterated collocation method.

The paper has been arranged in the following way. In Section 2 the notations are set and orders of convergence in the modified projection method with the orthogonal projection as well as the interpolatory projections are obtained. In Section 3 the projection is chosen to be either the orthogonal projection or the interpolatory projection at Gauss points, and the orders of convergence in the iterated modified projection are proved. Section 4 is devoted to the implementation details and numerical results are given in Section 5 .

2. Modified projection method. Let $X=L^{\infty}[0,1]$, and consider a Urysohn integral operator

$$
K(x)(s)=\int_{0}^{1} \kappa(s, t, x(t)) d t, \quad s \in[0,1], x \in X,
$$

where the kernel $\kappa(s, t, u)$ is a real valued continuous function. Let $\varphi$ be the unique solution of (1.1), and let $a$ and $b$ be real numbers such that

$$
\left[\min _{s \in[0,1]} \varphi(s), \max _{s \in[0,1]} \varphi(s)\right] \subset(a, b)
$$

Define

$$
\Omega=[0,1] \times[0,1] \times[a, b] .
$$

Let $\alpha \geq 1$. For $x \in C^{\alpha}[0,1]$, we define

$$
\|x\|_{\alpha, \infty}=\sum_{i=0}^{\alpha}\left\|x^{(i)}\right\|_{\infty}
$$

where $x^{(i)}$ denotes the $i$ th derivative of $x$. Assume that

$$
\kappa \in C^{\alpha}(\Omega) \text { and } \frac{\partial \kappa}{\partial u} \in C^{2 \alpha}(\Omega) .
$$


Then $K$ is a compact operator from $L^{\infty}[0,1]$ to $C^{\alpha}[0,1]$. As in Section 1, we assume that (1.1) has a unique solution $\varphi$. We also assume that $f \in C^{\alpha}[0,1]$. Then, since

$$
\varphi-K(\varphi)=f,
$$

the solution $\varphi$ of the above equation belongs to $C^{\alpha}[0,1]$.

The operator $K$ is Fréchet differentiable and the Fréchet derivative is given by

$$
\left(K^{\prime}(x) h\right)(s)=\int_{0}^{1} \frac{\partial \kappa}{\partial u}(s, t, x(t)) h(t) d t .
$$

Since by assumption,

$$
\frac{\partial \kappa}{\partial u} \in C^{2 \alpha}(\Omega)
$$

it follows that $K^{\prime}$ is Lipschitz continuous in a neighborhood $\mathcal{B}\left(\varphi, \delta_{0}\right)$ of $\varphi$, that is, there exists a constant $\gamma$ such that

$$
\left\|K^{\prime}(\varphi)-K^{\prime}(x)\right\| \leq \gamma\|\varphi-x\|_{\infty}, \quad x \in \mathcal{B}\left(\varphi, \delta_{0}\right) .
$$

The operator $K^{\prime}(\varphi)$ is compact. Assume that 1 is not an eigenvalue of $K^{\prime}(\varphi)$.

Let

$$
0=t_{0}^{(n)}<t_{1}^{(n)}<\cdots<t_{n}^{(n)}=1
$$

be a quasi-uniform partition of $[0,1]$. For $r \geq 0$, let $X_{n}$ denote the space of piecewise polynomials of degree $\leq r$ with respect to the above partition. For simplicity, we drop the index $n$ and write $t_{i}^{(n)}=t_{i}$, $i=0,1, \ldots, n$. Let

$$
h=\max \left\{t_{i}-t_{i-1}: i=1, \ldots, n\right\} \quad \text { and } \quad \beta=\min \{\alpha, r+1\} .
$$

We consider two types of projections from $L^{\infty}[0,1]$ to $X_{n}$.

1. The map $\pi_{n}$ is the restriction to $L^{\infty}[0,1]$ of the orthogonal projection from $L^{2}[0,1]$ to $X_{n}$.

2. Choose $r+1$ distinct points in each of the subinterval $\left[t_{i-1}, t_{i}\right]$, $i=1,2, \ldots, n$. 
Let $\pi_{n}: C[0,1] \rightarrow X_{n}$ be the map which interpolates a given function at $(r+1) n$ points in $[0,1]$. This map, if necessary, is extended to $L^{\infty}[0,1]$ as in Atkinson et al. [3] and then $\pi_{n}: L^{\infty}[0,1] \rightarrow X_{n}$ is a projection.

In both cases,

$$
\pi_{n} y \longrightarrow y, \quad y \in C[0,1]
$$

and, for $x \in C^{\beta}[0,1]$,

$$
\left\|x-\pi_{n} x\right\|_{\infty} \leq C_{1}\left\|x^{(\beta)}\right\|_{\infty} h^{\beta},
$$

where $C_{1}$ is a constant independent of $n$.

Thus, Theorem 1.1 is applicable with $f \neq 0$, and there exists a neighborhood $\mathcal{B}\left(\varphi, \delta_{0}\right)$ of $\varphi$, which contains, for all $n$ large enough, a unique solution $\varphi_{n}^{M}$ of (1.2).

The following result will be used in obtaining the order of convergence of $\varphi_{n}^{M}$ to $\varphi$.

Lemma 2.1. Let $X_{n}$ be the space of piecewise polynomials of degree $\leq r$ with respect to the partition (2.2), and let $\pi_{n}$ be either the restriction to $L^{\infty}[0,1]$ of the orthogonal projection from $L^{2}[0,1]$ to $X_{n}$ or an interpolatory projection from $L^{\infty}[0,1]$ to $X_{n}$. Then

$$
\left\|\left(I-\pi_{n}\right)\left[K\left(\pi_{n} \varphi\right)-K(\varphi)-K^{\prime}(\varphi)\left(\pi_{n} \varphi-\varphi\right)\right]\right\|_{\infty}=O\left(h^{3 \beta}\right) .
$$

Proof. Since by assumption,

$$
\frac{\partial \kappa}{\partial u} \in C^{2 \alpha}(\Omega) \quad \text { with } \alpha \geq 1
$$

it follows that for $v, w \in L^{\infty}[0,1]$,

$$
K^{\prime \prime}(\varphi)(v, w)(s)=\int_{0}^{1} \frac{\partial^{2} k}{\partial u^{2}}(s, t, \varphi(t)) v(t) w(t) d t
$$

The norm of the bilinear operator $K^{\prime \prime}(\varphi)$ is defined as follows:

$$
\left\|K^{\prime \prime}(\varphi)\right\|=\sup _{\|v\|_{\infty} \leq 1,\|w\|_{\infty} \leq 1}\left\|K^{\prime \prime}(\varphi)(v, w)\right\|_{\infty} .
$$


If $v \in \mathcal{B}\left(\varphi, \delta_{0}\right)$, then by Taylor's generalized theorem,

$$
\begin{aligned}
K(\varphi+v)(s)-K(\varphi)(s)- & K^{\prime}(\varphi) v(s) \\
& =\int_{0}^{1}(1-\theta)\left(K^{\prime \prime}(\varphi+\theta v) v^{2}\right)(s) d \theta \\
& :=(R v)(s), s \in[0,1] .
\end{aligned}
$$

Define

$$
\left(Q_{\theta} v\right)(s)=\left(K^{\prime \prime}(\varphi+\theta v) v^{2}\right)(s)=\int_{0}^{1} \frac{\partial^{2} k}{\partial u^{2}}(s, t, \varphi(t)+\theta v(t)) v^{2}(t) d t .
$$

Then

$$
(R v)(s)=\int_{0}^{1}(1-\theta)\left(Q_{\theta} v\right)(s) d \theta
$$

Since

$$
\frac{\partial \kappa}{\partial u} \in C^{2 \alpha}(\Omega)
$$

it follows that $Q_{\theta} v$, and hence $R v$, belong to $C^{2 \alpha-1}[0,1] \subset C^{\alpha}[0,1]$. We have

$$
\left(Q_{\theta} v\right)^{(\beta)}(s)=\int_{0}^{1} \frac{\partial^{\beta+2} k}{\partial s^{\beta} \partial u^{2}}(s, t, \varphi(t)+\theta v(t)) v^{2}(t) d t
$$

and

$$
(R v)^{(\beta)}(s)=\int_{0}^{1}(1-\theta)\left(Q_{\theta} v\right)^{(\beta)}(s) d \theta .
$$

Let

$$
C_{2}=\max _{\substack{s, t \in[0,1] \\|u| \leq\|\varphi\|_{\infty}+\delta_{0}}}\left|\frac{\partial^{\beta+2} k}{\partial s^{\beta} \partial u^{2}}(s, t, u)\right| .
$$

Then

$$
\left\|\left(Q_{\theta} v\right)^{(\beta)}\right\|_{\infty} \leq C_{2}\|v\|_{\infty}^{2}
$$

and

$$
\left\|(R v)^{(\beta)}\right\|_{\infty} \leq \frac{C_{2}}{2}\|v\|_{\infty}^{2}
$$


Since by $(2.3)$

$$
\left\|\left(I-\pi_{n}\right) R v\right\|_{\infty} \leq C_{1}\left\|(R v)^{(\beta)}\right\|_{\infty} h^{\beta}
$$

it follows that

$$
\left\|\left(I-\pi_{n}\right) R v\right\|_{\infty} \leq \frac{C_{1} C_{2}}{2}\|v\|_{\infty}^{2} h^{\beta} .
$$

Since $\pi_{n} y \rightarrow y, y \in C[0,1]$, for $n$ large enough, $\pi_{n} \varphi-\varphi \in B\left(\varphi, \delta_{0}\right)$. Hence,

$$
\left\|\left(I-\pi_{n}\right) R\left(\pi_{n} \varphi-\varphi\right)\right\|_{\infty} \leq \frac{C_{1} C_{2}}{2}\left\|\pi_{n} \varphi-\varphi\right\|_{\infty}^{2} h^{\beta}
$$

Since $\varphi \in C^{\alpha}[0,1]$, by $(2.3)$,

$$
\left\|\left(I-\pi_{n}\right) \varphi\right\|_{\infty} \leq C_{1}\left\|\varphi^{(\beta)}\right\|_{\infty} h^{\beta} .
$$

Thus,

$$
\begin{aligned}
&\left\|\left(I-\pi_{n}\right)\left[K\left(\pi_{n} \varphi\right)-K(\varphi)-K^{\prime}(\varphi)\left(\pi_{n} \varphi-\varphi\right)\right]\right\|_{\infty} \\
&=\left\|\left(I-\pi_{n}\right) R\left(\pi_{n} \varphi-\varphi\right)\right\|_{\infty} \\
& \leq \frac{C_{1}^{3} C_{2}}{2}\left\|\varphi^{(\beta)}\right\|_{\infty}^{2} h^{3 \beta}
\end{aligned}
$$

which completes the proof.

2.1. Orthogonal projection. In this section we consider $\pi_{n}$ : $L^{\infty}[0,1] \rightarrow X_{n}$ to be the restriction of the orthogonal projection from $L^{2}[0,1]$ to $X_{n}$ and obtain an error estimate for the approximate solution in the modified projection method. We first prove a preliminary result.

Lemma 2.2. For $\alpha \geq 1$, let $\kappa \in C^{\alpha}(\Omega), \frac{\partial \kappa}{\partial u} \in C^{2 \alpha}(\Omega)$ and $f \in C^{\alpha}[0,1]$. Let $\varphi$ be the unique solution of (1.1). Then

$$
\left\|\left(I-\pi_{n}\right) K^{\prime}(\varphi)\left(\pi_{n} \varphi-\varphi\right)\right\|_{\infty}=O\left(h^{3 \beta}\right) .
$$

Proof. Note that, for $v \in L^{\infty}[0,1]$,

$$
\begin{aligned}
& K^{\prime}(\varphi) v(s)=\int_{0}^{1} \frac{\partial k}{\partial u}(s, t, \varphi(t)) v(t) d t=\int_{0}^{1} \ell(s, t) v(t) d t \\
& s \in[0,1],
\end{aligned}
$$


with

$$
\ell(s, t)=\frac{\partial k}{\partial u}(s, t, \varphi(t)) .
$$

Since $\frac{\partial \kappa}{\partial u} \in C^{2 \alpha}(\Omega)$, it follows that

$$
K^{\prime}(\varphi) v \in C^{2 \alpha}([0,1])
$$

Hence, by (2.3),

$$
\left\|\left(I-\pi_{n}\right)\left(K^{\prime}(\varphi) v\right)\right\|_{\infty} \leq C_{1}\left\|\left(K^{\prime}(\varphi) v\right)^{(\beta)}\right\|_{\infty} h^{\beta} .
$$

Now

$$
\begin{aligned}
\left(K^{\prime}(\varphi) v\right)^{(\beta)}(s) & =\int_{0}^{1} \frac{\partial^{\beta+1} k}{\partial s^{\beta} \partial u}(s, t, \varphi(t)) v(t) d t \\
& =\int_{0}^{1} q(s, t) v(t) d t
\end{aligned}
$$

with

$$
q(s, t)=\frac{\partial^{\beta+1} k}{\partial s^{\beta} \partial u}(s, t, \varphi(t)), \quad s, t \in[0,1] .
$$

For a fixed $s \in[0,1]$, define

$$
q_{s}(t)=q(s, t), \quad t \in[0,1] .
$$

Then, as $\beta=\min \{\alpha, r+1\}$,

$$
q_{s} \in C^{2 \alpha-\beta}[0,1] \subset C^{\alpha}[0,1] .
$$

Using the fact that $\pi_{n}$ is the restriction to $L^{\infty}[0,1]$ of the orthogonal projection from $L^{2}[0,1]$ to $X_{n}$, we obtain

$$
\begin{aligned}
\left(K^{\prime}(\varphi)\left(\pi_{n} \varphi-\varphi\right)\right)^{(\beta)}(s) & =\int_{0}^{1} q(s, t)\left(\pi_{n} \varphi-\varphi\right)(t) d t \\
& =\left\langle q_{s},\left(\pi_{n}-I\right) \varphi\right\rangle \\
& =-\left\langle\left(\pi_{n}-I\right) q_{s},\left(\pi_{n}-I\right) \varphi\right\rangle .
\end{aligned}
$$

Let

$$
C_{3}=\max _{s, t \in[0,1]}\left|\frac{\partial^{2 \beta+1} k}{\partial s^{\beta} \partial t^{\beta} \partial u}(s, t, \varphi(t))\right| .
$$


Then

$$
\left\|\left(\pi_{n}-I\right) q_{s}\right\|_{\infty} \leq C_{1}\left\|\left(q_{s}\right)^{(\beta)}\right\|_{\infty} h^{\beta} \leq\left(C_{1} C_{3}\right) h^{\beta} .
$$

Since $\varphi \in C^{\alpha}[0,1]$,

$$
\left\|\left(\pi_{n}-I\right) \varphi\right\|_{\infty} \leq C_{1}\left\|\varphi^{(\beta)}\right\|_{\infty} h^{\beta} .
$$

Thus, for each $s \in[0,1]$,

$$
\left|\left(K^{\prime}(\varphi)\left(\pi_{n} \varphi-\varphi\right)\right)^{(\beta)}(s)\right| \leq C_{1}^{2} C_{3}\left\|\varphi^{(\beta)}\right\|_{\infty} h^{2 \beta}
$$

and hence

$$
\left\|\left(K^{\prime}(\varphi)\left(\pi_{n} \varphi-\varphi\right)\right)^{(\beta)}\right\|_{\infty} \leq C_{1}^{2} C_{3}\left\|\varphi^{(\beta)}\right\|_{\infty} h^{2 \beta} .
$$

From (2.7), we then obtain

$$
\begin{aligned}
\left\|\left(I-\pi_{n}\right) K^{\prime}(\varphi)\left(\pi_{n} \varphi-\varphi\right)\right\|_{\infty} \leq C_{1} \|\left(K^{\prime}(\varphi)\right. & \left.\left(\pi_{n} \varphi-\varphi\right)\right)^{(\beta)} \|_{\infty} h^{\beta} \\
& \leq C_{1}^{3} C_{3}\left\|\varphi^{(\beta)}\right\|_{\infty} h^{3 \beta}
\end{aligned}
$$

which proves the result.

Theorem 2.3. For $\alpha \geq 1$, let $\kappa \in C^{\alpha}(\Omega)$,

$$
\frac{\partial \kappa}{\partial u} \in C^{2 \alpha}(\Omega) \quad \text { and } \quad f \in C^{\alpha}[0,1]
$$

Let $\varphi$ be the unique solution of (1.1), and assume that 1 is not an eigenvalue of $K^{\prime}(\varphi)$. Let $X_{n}$ be the space of piecewise polynomials of degree $\leq r$ with respect to the partition $(2.2)$ and $\pi_{n}: L^{\infty}[0,1] \rightarrow X_{n}$ the restriction of the orthogonal projection from $L^{2}[0,1]$ to $X_{n}$. Let $\varphi_{n}^{M}$ be the unique solution of $(1.2)$ in $B\left(\varphi, \delta_{0}\right)$. Then

$$
\left\|\varphi_{n}^{M}-\varphi\right\|_{\infty}=O\left(h^{3 \beta}\right)
$$

Proof. By assumption, $I-K^{\prime}(\varphi)$ is invertible. From (1.3),

$$
\left(K_{n}^{M}\right)^{\prime}(\varphi)=\pi_{n} K^{\prime}(\varphi)+\left(I-\pi_{n}\right) K^{\prime}\left(\pi_{n} \varphi\right) \pi_{n}
$$


Hence,

$K^{\prime}(\varphi)-\left(K_{n}^{M}\right)^{\prime}(\varphi)=\left(I-\pi_{n}\right) K^{\prime}(\varphi)\left(I-\pi_{n}\right)+\left(I-\pi_{n}\right)\left(K^{\prime}(\varphi)-K^{\prime}\left(\pi_{n} \varphi\right)\right) \pi_{n}$.

Since $\pi_{n} \rightarrow I$ pointwise on $C[0,1]$ and $K^{\prime}(\varphi): L^{\infty}[0,1] \rightarrow C[0,1]$ is compact, it follows that

$$
\left\|\left(I-\pi_{n}\right) K^{\prime}(\varphi)\right\| \longrightarrow 0 \quad \text { as } n \rightarrow \infty .
$$

By (2.1),

$$
\left\|K^{\prime}(\varphi)-K^{\prime}\left(\pi_{n} \varphi\right)\right\| \leq \gamma\left\|\varphi-\pi_{n} \varphi\right\|_{\infty} \longrightarrow 0 \quad \text { as } n \rightarrow \infty .
$$

Thus, since the sequence $\left(\left\|\pi_{n}\right\|\right)$ is uniformly bounded,

$$
\left\|K^{\prime}(\varphi)-\left(K_{n}^{M}\right)^{\prime}(\varphi)\right\| \longrightarrow 0 \quad \text { as } n \rightarrow \infty .
$$

It follows that, for all $n$ large enough, $I-\left(K_{n}^{M}\right)^{\prime}(\varphi)$ is invertible and

$$
\left\|\left(I-\left(K_{n}^{M}\right)^{\prime}(\varphi)\right)^{-1}\right\| \leq 2\left\|\left(I-K^{\prime}(\varphi)\right)^{-1}\right\| .
$$

By [7, Theorem 1.1], we have

$$
\left\|\varphi_{n}^{M}-\varphi\right\|_{\infty} \leq 2 \alpha_{n}
$$

where

$$
\begin{aligned}
\alpha_{n} & =\left\|\left(I-\left(K_{n}^{M}\right)^{\prime}(\varphi)\right)^{-1}\left[K(\varphi)-K_{n}^{M}(\varphi)\right]\right\|_{\infty} \\
& =\left\|\left(I-\left(K_{n}^{M}\right)^{\prime}(\varphi)\right)^{-1}\left[\left(I-\pi_{n}\right)\left(K(\varphi)-K\left(\pi_{n} \varphi\right)\right)\right]\right\|_{\infty} .
\end{aligned}
$$

Hence,

(2.10) $\left\|\varphi_{n}^{M}-\varphi\right\|_{\infty} \leq 4\left\|\left(I-K^{\prime}(\varphi)\right)^{-1}\right\|\left\|\left(I-\pi_{n}\right)\left(K(\varphi)-K\left(\pi_{n} \varphi\right)\right)\right\|_{\infty}$.

Consider

$$
\begin{aligned}
\left(I-\pi_{n}\right)\left(K(\varphi)-K\left(\pi_{n} \varphi\right)\right) & \\
= & -\left(I-\pi_{n}\right)\left[K\left(\pi_{n} \varphi\right)-K(\varphi)-K^{\prime}(\varphi)\left(\pi_{n} \varphi-\varphi\right)\right] \\
& -\left(I-\pi_{n}\right) K^{\prime}(\varphi)\left(\pi_{n} \varphi-\varphi\right) .
\end{aligned}
$$


By Lemma 2.1,

$$
\left\|\left(I-\pi_{n}\right)\left[K\left(\pi_{n} \varphi\right)-K(\varphi)-K^{\prime}(\varphi)\left(\pi_{n} \varphi-\varphi\right)\right]\right\|_{\infty}=O\left(h^{3 \beta}\right)
$$

and by Lemma 2.2,

$$
\left\|\left(I-\pi_{n}\right) K^{\prime}(\varphi)\left(\pi_{n} \varphi-\varphi\right)\right\|_{\infty}=O\left(h^{3 \beta}\right)
$$

Combining (2.10), (2.11) and the above two estimates, we obtain the desired result.

2.2. Interpolatory projection. Let $\tau_{0}, \tau_{1}, \ldots, \tau_{r}$ be $r+1$ distinct points in $[-1,1]$. Consider the partition $(2.2)$ of $[0,1]$ and, for $i=$ $1, \ldots, n$, define a function

$$
f_{i}(t)=\frac{1-t}{2} t_{i-1}+\frac{1+t}{2} t_{i}, \quad t \in[-1,1] .
$$

Then $f_{i}:[-1,1] \rightarrow\left[t_{i-1}, t_{i}\right]$ is a one-to-one, onto and affine map. Let

$$
S=\left\{\tau_{i, j}=f_{i}\left(\tau_{j}\right), \quad i=1, \ldots, n, j=0,1, \ldots, r\right\}
$$

be the set of collocation points and $\pi_{n}: C[0,1] \rightarrow X_{n}$ defined by

$$
\left(\pi_{n} x\right)\left(\tau_{i, j}\right)=x\left(\tau_{i, j}\right), \quad i=1, \ldots, n, j=0,1, \ldots, r .
$$

If $\tau_{0}=-1$ and $\tau_{r}=1$, then $\pi_{n} x$ is continuous, and we can choose $X_{n}$ to be the space of continuous piecewise polynomials of degree $\leq r$ with respect to the partition (2.2). Thus, $X_{n} \subset C[0,1]$ and $\pi_{n}: C[0,1] \rightarrow X_{n}$ is a projection.

Otherwise, since $\pi_{n} x$ is not necessarily continuous, we need to choose $X_{n}$ to be the space of discontinuous piecewise polynomials of degree $\leq r$ with respect to the partition (2.2). In this case, $X_{n} \subset L^{\infty}[0,1]$, and we extend $\pi_{n}$ to $L^{\infty}[0,1]$ so that $\pi_{n}: L^{\infty}[0,1] \rightarrow X_{n}$ is a projection. (See Atkinson et al. [3].)

Theorem 2.4. For $\alpha \geq 1$, let $\kappa \in C^{\alpha}(\Omega)$, $\frac{\partial \kappa}{\partial u} \in C^{2 \alpha}(\Omega)$ and $f \in C^{\alpha}[0,1]$. Let $\varphi$ be the unique solution of $(1.1)$, and assume that 1 is not an eigenvalue of $K^{\prime}(\varphi)$. Let $X_{n}$ be the space of piecewise polynomials of degree $\leq r$ with respect to the partition (2.2), and let 
$\pi_{n}: L^{\infty}[0,1] \rightarrow X_{n}$ be an interpolatory projection. Let $\varphi_{n}^{M}$ be the unique solution of $(1.2)$ in $B\left(\varphi, \delta_{0}\right)$. Then

$$
\left\|\varphi_{n}^{M}-\varphi\right\|_{\infty}=O\left(h^{2 \beta}\right) .
$$

Proof. Recall from (2.10) that

$$
\left\|\varphi_{n}^{M}-\varphi\right\|_{\infty} \leq 4\left\|\left(I-K^{\prime}(\varphi)\right)^{-1}\right\|\left\|\left(I-\pi_{n}\right)\left(K(\varphi)-K\left(\pi_{n} \varphi\right)\right)\right\|_{\infty} .
$$

As before, we write

$$
\begin{aligned}
\left(I-\pi_{n}\right)(K(\varphi)- & \left.K\left(\pi_{n} \varphi\right)\right) \\
= & -\left(I-\pi_{n}\right)\left[K\left(\pi_{n} \varphi\right)-K(\varphi)-K^{\prime}(\varphi)\left(\pi_{n} \varphi-\varphi\right)\right] \\
& -\left(I-\pi_{n}\right) K^{\prime}(\varphi)\left(\pi_{n} \varphi-\varphi\right) .
\end{aligned}
$$

By Lemma 2.1, we have

$$
\left\|\left(I-\pi_{n}\right)\left[K\left(\pi_{n} \varphi\right)-K(\varphi)-K^{\prime}(\varphi)\left(\pi_{n} \varphi-\varphi\right)\right]\right\|_{\infty}=O\left(h^{3 \beta}\right) .
$$

On the other hand, by $(2.3)$

$$
\left\|\left(I-\pi_{n}\right) K^{\prime}(\varphi)\left(\pi_{n} \varphi-\varphi\right)\right\|_{\infty} \leq C_{1}\left\|\left(K^{\prime}(\varphi)\left(\pi_{n} \varphi-\varphi\right)\right)^{(\beta)}\right\|_{\infty} h^{\beta} .
$$

Recall from (2.8) that

$$
\left(K^{\prime}(\varphi)\left(\pi_{n} \varphi-\varphi\right)\right)^{(\beta)}(s)=\int_{0}^{1} q(s, t)\left(\pi_{n} \varphi-\varphi\right)(t) d t
$$

Hence,

$$
\left\|\left(K^{\prime}(\varphi)\left(\pi_{n} \varphi-\varphi\right)\right)^{(\beta)}\right\|_{\infty} \leq\|q\|_{\infty}\left\|\pi_{n} \varphi-\varphi\right\|_{\infty} \leq C_{1}\|q\|_{\infty}\left\|\varphi^{(\beta)}\right\|_{\infty} h^{\beta} .
$$

Thus,

$$
\left\|\left(I-\pi_{n}\right) K^{\prime}(\varphi)\left(\pi_{n} \varphi-\varphi\right)\right\|_{\infty} \leq\left(C_{1}\right)^{2}\|q\|_{\infty}\left\|\varphi^{(\beta)}\right\|_{\infty} h^{2 \beta} .
$$

From $(2.13),(2.14)$ and the above estimate, we obtain

$$
\left\|\varphi_{n}^{M}-\varphi\right\|_{\infty}=O\left(h^{2 \beta}\right)
$$


Remark 2.5. It is known that in the present case the error in the collocation method is of the order of $\beta$ and one step of iteration does not improve this order of convergence. (See [4].) However, the above result shows that the error in the modified projection method is of the order of $2 \beta$, and thus the approximate solution in the modified projection improves upon the approximate solutions in the collocation/iterated collocation methods.

We now consider the collocation at the Gauss points. Choose $\tau_{0}, \tau_{1}, \ldots, \tau_{r}$ in $[-1,1]$ to be the zeroes of the Legendre polynomial of degree $r+1$, and let

$$
\left\{\tau_{i, j}=f_{i}\left(\tau_{j}\right), \quad i=1, \ldots, n, j=0,1, \ldots, r\right\}
$$

be the set of collocation points. Let $X_{n}$ be the space of discontinuous piecewise polynomials of degree $\leq r$ with respect to the partition (2.2), and let $\pi_{n}: C[0,1] \rightarrow X_{n}$ be defined by

$$
\left(\pi_{n} x\right)\left(\tau_{i, j}\right)=x\left(\tau_{i, j}\right), \quad i=1, \ldots, n, j=0,1, \ldots, r .
$$

We quote the following estimate of de Boor and Swartz [6]: For $x \in C^{\beta}[0,1]$ and $y \in C^{2 \beta}[0,1]$,

$$
\left|\int_{0}^{1} x(t)\left(I-\pi_{n}\right) y(t) d t\right| \leq C_{4}\|x\|_{\beta, \infty}\|y\|_{2 \beta, \infty} h^{2 \beta},
$$

where $C_{4}$ is a constant independent of $h$.

In the present case we need to make the following stronger assumptions:

$$
\kappa, \frac{\partial \kappa}{\partial u} \in C^{2 \alpha}(\Omega) \quad \text { and } \quad f \in C^{2 \alpha}[0,1] .
$$

Then $\varphi \in C^{2 \alpha}[0,1]$. Using estimate (2.16), we can improve the order of convergence $\beta$ in (2.15) to $2 \beta$ and obtain the following improved estimate.

Theorem 2.6. For $\alpha \geq 1$, let $\kappa, \frac{\partial \kappa}{\partial u} \in C^{2 \alpha}(\Omega)$ and $f \in C^{2 \alpha}[0,1]$. Let $\varphi$ be the unique solution of (1.1), and assume that 1 is not an eigenvalue of $K^{\prime}(\varphi)$. Let $X_{n}$ be the space of piecewise polynomials of degree $\leq r$ 
with respect to the partition (2.2), and let $\pi_{n}: L^{\infty}[0,1] \rightarrow X_{n}$ be the interpolatory projection at $r+1$ Gauss points. Let $\varphi_{n}^{M}$ be the unique solution of $(1.2)$ in $B\left(\varphi, \delta_{0}\right)$. Then

$$
\left\|\varphi_{n}^{M}-\varphi\right\|_{\infty}=O\left(h^{3 \beta}\right) .
$$

Proof. From (2.10)

$$
\begin{aligned}
\left\|\varphi_{n}^{M}-\varphi\right\|_{\infty} \leq & 4\left\|\left(I-K^{\prime}(\varphi)\right)^{-1}\right\|\left\|\left(I-\pi_{n}\right)\left(K(\varphi)-K\left(\pi_{n} \varphi\right)\right)\right\|_{\infty} \\
\leq & 4\left\|\left(I-K^{\prime}(\varphi)\right)^{-1}\right\| \|\left(I-\pi_{n}\right) \\
& \times\left[K\left(\pi_{n} \varphi\right)-K(\varphi)-K^{\prime}(\varphi)\left(\pi_{n} \varphi-\varphi\right)\right] \|_{\infty} \\
& +4\left\|\left(I-K^{\prime}(\varphi)\right)^{-1}\right\|\left\|\left(I-\pi_{n}\right) K^{\prime}(\varphi)\left(\pi_{n} \varphi-\varphi\right)\right\|_{\infty} .
\end{aligned}
$$

Note that estimate (2.14) is still valid, and we have

$$
\left\|\left(I-\pi_{n}\right)\left[K\left(\pi_{n} \varphi\right)-K(\varphi)-K^{\prime}(\varphi)\left(\pi_{n} \varphi-\varphi\right)\right]\right\|_{\infty}=O\left(h^{3 \beta}\right) .
$$

By $(2.3)$,

$$
\left\|\left(I-\pi_{n}\right) K^{\prime}(\varphi)\left(\pi_{n} \varphi-\varphi\right)\right\|_{\infty} \leq C_{1}\left\|\left(K^{\prime}(\varphi)\left(\pi_{n} \varphi-\varphi\right)\right)^{(\beta)}\right\|_{\infty} h^{\beta} .
$$

From (2.8),

$$
\left(K^{\prime}(\varphi)\left(\pi_{n} \varphi-\varphi\right)\right)^{(\beta)}(s)=\int_{0}^{1} \frac{\partial^{\beta+1} k}{\partial s^{\beta} \partial u}(s, t, \varphi(t))\left(\pi_{n} \varphi-\varphi\right)(t) d t .
$$

Let

$$
C_{5}=\sum_{j=0}^{\beta} \max _{s, t \in[0,1]}\left|\frac{\partial^{\beta+j+1} k}{\partial s^{\beta} \partial t^{j} \partial u}(s, t, \varphi(t))\right| .
$$

For $s \in[0,1]$, using estimate $(2.16)$, we obtain

$$
\left|\left(K^{\prime}(\varphi)\left(\pi_{n} \varphi-\varphi\right)\right)^{(\beta)}(s)\right| \leq C_{4} C_{5}\|\varphi\|_{2 \beta, \infty} h^{2 \beta} .
$$

Hence,

$$
\left\|\left(I-\pi_{n}\right) K^{\prime}(\varphi)\left(\pi_{n} \varphi-\varphi\right)\right\|_{\infty} \leq C_{1} C_{4} C_{5}\|\varphi\|_{2 \beta, \infty} h^{3 \beta},
$$


and it follows that

$$
\left\|\varphi_{n}^{M}-\varphi\right\|_{\infty}=O\left(h^{3 \beta}\right)
$$

Remark 2.7. In the case when the kernel of $K$ is sufficiently smooth, that is, $\alpha \geq r+1$, we have

$$
\beta=\min \{\alpha, r+1\}=r+1
$$

Hence, we obtain the following estimates. If $\pi_{n}$ is either the orthogonal projection or the interpolatory projection at Gauss points, then by Theorem 2.3 and by Theorem 2.6, respectively,

$$
\left\|\varphi_{n}^{M}-\varphi\right\|_{\infty}=O\left(h^{3 r+3}\right)
$$

whereas if the collocation points are not the Gauss points, then by Theorem 2.4,

$$
\left\|\varphi_{n}^{M}-\varphi\right\|_{\infty}=O\left(h^{2 r+2}\right) .
$$

3. Improvement by iteration. Let $\varphi_{n}^{M}$ be the unique solution of $(1.2)$ in $B\left(\varphi, \delta_{0}\right)$, that is,

$$
\varphi_{n}^{M}-K_{n}^{M}\left(\varphi_{n}^{M}\right)=f
$$

Define

$$
\widetilde{\varphi}_{n}^{M}=K\left(\varphi_{n}^{M}\right)+f
$$

If $\pi_{n}$ is either the orthogonal projection or the interpolatory projection at the Gauss points, then we show that this one step of iteration improves the order of convergence from $3 \beta$ to $4 \beta$.

Throughout this section, we make the following assumptions.

Let $X_{n}$ be the space of piecewise polynomials of degree $\leq r$ with respect to the partition (2.2). 
If $\pi_{n}$ is the orthogonal projection onto $X_{n}$, then we assume that

$$
\kappa \in C^{\alpha}(\Omega), \frac{\partial \kappa}{\partial u} \in C^{2 \alpha}(\Omega) \text { and } f \in C^{\alpha}[0,1] .
$$

On the other hand, if $\pi_{n}$ is the interpolatory projection at Gauss points, then we assume that

$$
\kappa, \frac{\partial \kappa}{\partial u} \in C^{2 \alpha}(\Omega) \quad \text { and } \quad f \in C^{2 \alpha}[0,1] .
$$

We first prove some preliminary results.

Lemma 3.1. Let $\pi_{n}$ be either the orthogonal projection or the interpolatory projection at $r+1$ Gauss points with the range equal to $X_{n}$. Then

$$
\left\|K^{\prime}(\varphi)\left(I-\pi_{n}\right)\left[K\left(\pi_{n} \varphi\right)-K(\varphi)-K^{\prime}(\varphi)\left(\pi_{n} \varphi-\varphi\right)\right]\right\|_{\infty}=O\left(h^{4 \beta}\right) .
$$

Proof. Let

$$
x_{n}=K\left(\pi_{n} \varphi\right)-K(\varphi)-K^{\prime}(\varphi)\left(\pi_{n} \varphi-\varphi\right) .
$$

From (2.6),

$$
K^{\prime}(\varphi)\left(I-\pi_{n}\right) x_{n}(s)=\int_{0}^{1} \ell(s, t)\left(I-\pi_{n}\right) x_{n}(t) d t, \quad s \in[0,1],
$$

where

$$
\ell(s, t)=\frac{\partial k}{\partial u}(s, t, \varphi(t)) .
$$

For a fixed $s \in[0,1]$, let

$$
\ell_{s}(t)=\ell(s, t), \quad t \in[0,1] .
$$

If $\pi_{n}$ is the orthogonal projection, then

$$
\begin{aligned}
K^{\prime}(\varphi)\left(I-\pi_{n}\right) x_{n}(s) & =\left\langle l_{s},\left(I-\pi_{n}\right) x_{n}\right\rangle \\
& =\left\langle\left(I-\pi_{n}\right) l_{s},\left(I-\pi_{n}\right) x_{n}\right\rangle .
\end{aligned}
$$


Hence, using (2.3), we obtain

$$
\left|K^{\prime}(\varphi)\left(I-\pi_{n}\right) x_{n}(s)\right| \leq\left(C_{1}\right)^{2}\left\|\left(l_{s}\right)^{(\beta)}\right\|_{\infty}\left\|\left(x_{n}\right)^{(\beta)}\right\|_{\infty} h^{2 \beta}, \quad s \in[0,1] .
$$

Let

$$
C_{6}=\max _{s, t \in[0,1]}\left|\frac{\partial^{\beta+1} k}{\partial t^{\beta} \partial u}(s, t, \varphi(t))\right| .
$$

Then

$$
\left\|\left(l_{s}\right)^{(\beta)}\right\|_{\infty} \leq C_{6}
$$

and

$$
\left\|K^{\prime}(\varphi)\left(I-\pi_{n}\right) x_{n}\right\|_{\infty} \leq\left(C_{1}\right)^{2} C_{6}\left\|\left(x_{n}\right)^{(\beta)}\right\|_{\infty} h^{2 \beta} .
$$

Recall from (2.4) that for $n$ large enough,

$$
x_{n}=K\left(\pi_{n} \varphi\right)-K(\varphi)-K^{\prime}(\varphi)\left(\pi_{n} \varphi-\varphi\right)=R\left(\pi_{n} \varphi-\varphi\right) .
$$

From (2.5) we obtain

$$
\left\|\left(x_{n}\right)^{(\beta)}\right\|_{\infty}=\left\|\left(R\left(\pi_{n} \varphi-\varphi\right)\right)^{(\beta)}\right\|_{\infty} \leq \frac{C_{2}}{2}\left\|\pi_{n} \varphi-\varphi\right\|_{\infty}^{2} .
$$

Thus,

$$
\left\|\left(x_{n}\right)^{(\beta)}\right\|_{\infty} \leq \frac{\left(C_{1}\right)^{2} C_{2}}{2}\left\|\varphi^{(\beta)}\right\|_{\infty}^{2} h^{2 \beta}
$$

and

$$
\left\|K^{\prime}(\varphi)\left(I-\pi_{n}\right) x_{n}\right\|_{\infty} \leq \frac{\left(C_{1}\right)^{4} C_{2} C_{6}}{2}\left\|\varphi^{(\beta)}\right\|_{\infty}^{2} h^{4 \beta}
$$

which proves (3.2) in the case of the orthogonal projection.

If $\pi_{n}$ is the interpolatory projection at $r+1$ Gauss points, then using (2.16) we obtain

$$
\begin{aligned}
\left|K^{\prime}(\varphi)\left(I-\pi_{n}\right) x_{n}(s)\right| & =\left|\int_{0}^{1} \ell(s, t)\left(I-\pi_{n}\right) x_{n}(t) d t\right| \\
& \leq C_{4}\left\|l_{s}\right\|_{\beta, \infty}\left\|x_{n}\right\|_{2 \beta, \infty} h^{2 \beta} .
\end{aligned}
$$

Let

$$
C_{7}=\sum_{j=0}^{\beta} \max _{s, t \in[0,1]}\left|\frac{\partial^{j+1} k}{\partial t^{j} \partial u}(s, t, \varphi)\right|
$$


Then, for $s \in[0,1]$,

$$
\left\|l_{s}\right\|_{\beta, \infty} \leq C_{7} .
$$

It can easily be checked that

$$
\begin{aligned}
\left\|x_{n}\right\|_{2 \beta, \infty} & =\sum_{j=0}^{2 \beta}\left\|\left(x_{n}\right)^{(j)}\right\|_{\infty} \\
& =\sum_{j=0}^{2 \beta}\left\|\left(R\left(\pi_{n} \varphi-\varphi\right)\right)^{(j)}\right\|_{\infty} \\
& \leq \frac{C_{8}}{2}\left\|\pi_{n} \varphi-\varphi\right\|_{\infty}^{2} \\
& \leq \frac{\left(C_{1}\right)^{2} C_{8}}{2}\left\|\varphi^{(\beta)}\right\|_{\infty}^{2} h^{2 \beta}
\end{aligned}
$$

where

$$
C_{8}=\sum_{j=0}^{2 \beta} \max _{\substack{s, t \in[0,1] \\|u| \leq\|\varphi\|_{\infty}+\delta_{0}}}\left|\frac{\partial^{j+2} k}{\partial s^{j} \partial u^{2}}(s, t, u)\right| .
$$

Combining (3.3), (3.4) and (3.5), we obtain

$$
\left\|K^{\prime}(\varphi)\left(I-\pi_{n}\right) x_{n}\right\|_{\infty} \leq \frac{\left(C_{1}\right)^{2} C_{4} C_{7} C_{8}}{2}\left\|\varphi^{(\beta)}\right\|_{\infty}^{2} h^{4 \beta},
$$

which completes the proof.

Lemma 3.2. Let $\pi_{n}$ be either the orthogonal projection or the interpolatory projection at $r+1$ Gauss points with the range equal to $X_{n}$. Then

$$
\left\|K^{\prime}(\varphi)\left[K(\varphi)-K_{n}^{M}(\varphi)\right]\right\|_{\infty}=O\left(h^{4 \beta}\right) .
$$

Proof. Since by definition

$$
K_{n}^{M}(\varphi)=\pi_{n} K(\varphi)+K\left(\pi_{n} \varphi\right)-\pi_{n} K\left(\pi_{n} \varphi\right),
$$

we have

$$
\begin{aligned}
K(\varphi)-K_{n}^{M}(\varphi)= & \left(I-\pi_{n}\right)\left(K(\varphi)-K\left(\pi_{n} \varphi\right)\right) \\
= & -\left(I-\pi_{n}\right)\left[K\left(\pi_{n} \varphi\right)-K(\varphi)-K^{\prime}(\varphi)\left(\pi_{n} \varphi-\varphi\right)\right] \\
& -\left(I-\pi_{n}\right) K^{\prime}(\varphi)\left(\pi_{n} \varphi-\varphi\right) .
\end{aligned}
$$


Hence,

$$
\begin{aligned}
\| K^{\prime}(\varphi)[ & \left.K(\varphi)-K_{n}^{M}(\varphi)\right] \|_{\infty} \\
\leq & \left\|K^{\prime}(\varphi)\left(I-\pi_{n}\right)\left[K\left(\pi_{n} \varphi\right)-K(\varphi)-K^{\prime}(\varphi)\left(\pi_{n} \varphi-\varphi\right)\right]\right\|_{\infty} \\
& +\left\|K^{\prime}(\varphi)\left(I-\pi_{n}\right) K^{\prime}(\varphi)\left(\pi_{n} \varphi-\varphi\right)\right\|_{\infty} .
\end{aligned}
$$

By Lemma 3.1,

$$
\left\|K^{\prime}(\varphi)\left(I-\pi_{n}\right)\left[K\left(\pi_{n} \varphi\right)-K(\varphi)-K^{\prime}(\varphi)\left(\pi_{n} \varphi-\varphi\right)\right]\right\|_{\infty}=O\left(h^{4 \beta}\right) .
$$

Note that $K^{\prime}(\varphi)$ is a linear integral operator with a kernel $\ell \in$ $C^{2 \alpha}([0,1] \times[0,1])$. In the case of orthogonal projection, by estimate (4.6) of [12, Proposition 4.2], we obtain

$$
\left\|K^{\prime}(\varphi)\left(I-\pi_{n}\right) K^{\prime}(\varphi)\left(\pi_{n} \varphi-\varphi\right)\right\|_{\infty}=O\left(h^{4 \beta}\right) .
$$

In the case of the interpolatory projection at Gauss points, the above estimate is obtained from [12, Proposition 4.4]. Hence,

$$
\left\|K^{\prime}(\varphi)\left[K(\varphi)-K_{n}^{M}(\varphi)\right]\right\|_{\infty}=O\left(h^{4 \beta}\right)
$$

which completes the proof.

Lemma 3.3. Let $\pi_{n}$ be either the orthogonal projection or the interpolatory projection at $r+1$ Gauss points with the range equal to $X_{n}$. Then

$$
\left\|K_{n}^{M}\left(\varphi_{n}^{M}\right)-K_{n}^{M}(\varphi)-\left(K_{n}^{M}\right)^{\prime}(\varphi)\left(\varphi_{n}^{M}-\varphi\right)\right\|_{\infty}=O\left(h^{6 \beta}\right) .
$$

Proof. If $v \in B\left(\varphi, \delta_{0}\right)$, then by Taylor's generalized theorem,

$$
\begin{aligned}
K_{n}^{M}(\varphi+v)(s)-K_{n}^{M}(\varphi)(s) & -\left(K_{n}^{M}\right)^{\prime}(\varphi) v(s) \\
= & \int_{0}^{1}(1-\theta)\left(\left(K_{n}^{M}\right)^{\prime \prime}(\varphi+\theta v) v^{2}\right)(s) d \theta .
\end{aligned}
$$

Hence,

$$
\begin{aligned}
\| K_{n}^{M}\left(\varphi_{n}^{M}\right) & -K_{n}^{M}(\varphi)-\left(K_{n}^{M}\right)^{\prime}(\varphi)\left(\varphi_{n}^{M}-\varphi\right) \|_{\infty} \\
& \leq \frac{1}{2} \max _{0 \leq \theta \leq 1}\left\|\left(K_{n}^{M}\right)^{\prime \prime}\left(\varphi+\theta\left(\varphi_{n}^{M}-\varphi\right)\right)\right\|\left\|\varphi_{n}^{M}-\varphi\right\|_{\infty}^{2} .
\end{aligned}
$$


Note that, since $\pi_{n}$ is a linear map,

$$
\left(K_{n}^{M}\right)^{\prime \prime}(x)=\pi_{n} K^{\prime \prime}(x)+\left(I-\pi_{n}\right) K^{\prime \prime}\left(\pi_{n} x\right)\left(\pi_{n} \otimes \pi_{n}\right),
$$

where $\pi_{n} \otimes \pi_{n}: X \times X \rightarrow X \times X$ is defined as

$$
\left(\pi_{n} \otimes \pi_{n}\right)(v, w)=\left(\pi_{n} v, \pi_{n} w\right) .
$$

Let

$$
C_{9}=\max _{\substack{s, t \in[0,1] \\|u| \leq\|\varphi\|_{\infty}+\delta_{0}}}\left|\frac{\partial^{2} k}{\partial u^{2}}(s, t, u)\right| .
$$

Then, since

$$
\begin{aligned}
& K^{\prime \prime}\left(\varphi+\theta\left(\varphi_{n}^{M}-\varphi\right)\right)(v, w)(s) \\
&=\int_{0}^{1} \frac{\partial^{2} k}{\partial u^{2}}\left(s, t, \varphi(t)+\theta\left(\varphi_{n}^{M}(t)-\varphi(t)\right)\right) v(t) w(t) d t,
\end{aligned}
$$

it follows that, for $0 \leq \theta \leq 1$ and for $n$ large enough,

$$
\left\|K^{\prime \prime}\left(\varphi+\theta\left(\varphi_{n}^{M}-\varphi\right)\right)\right\| \leq C_{9} .
$$

In a similar manner, for $0 \leq \theta \leq 1$ and for $n$ large enough,

$$
\left\|K^{\prime \prime}\left(\pi_{n} \varphi+\theta\left(\pi_{n} \varphi_{n}^{M}-\pi_{n} \varphi\right)\right)\right\| \leq C_{9} .
$$

Since the sequence $\left(\left\|\pi_{n}\right\|\right)$ is uniformly bounded, it follows that

$$
\max _{0 \leq \theta \leq 1}\left\|\left(K_{n}^{M}\right)^{\prime \prime}\left(\varphi+\theta\left(\varphi_{n}^{M}-\varphi\right)\right)\right\| \leq C_{10},
$$

where $C_{10}$ is a constant independent of $n$.

By Theorem 2.3 in the case of the orthogonal projection and by Theorem 2.6 in the case of the interpolatory projection at Gauss points, we have

$$
\left\|\varphi_{n}^{M}-\varphi\right\|_{\infty}=O\left(h^{3 \beta}\right),
$$

and thus the desired result follows from (3.6). 
Lemma 3.4. Let $\pi_{n}$ be either the orthogonal projection or the interpolatory projection at $r+1$ Gauss points with the range equal to $X_{n}$. Then

$$
\left\|K^{\prime}(\varphi)\left(\left(K_{n}^{M}\right)^{\prime}(\varphi)-K^{\prime}(\varphi)\right)\left(\varphi-\varphi_{n}^{M}\right)\right\|_{\infty}=O\left(h^{4 \beta}\right)
$$

Proof. Note that

$$
\begin{aligned}
& K^{\prime}(\varphi)\left(\left(K_{n}^{M}\right)^{\prime}(\varphi)-K^{\prime}(\varphi)\right) \\
& \quad=-K^{\prime}(\varphi)\left(I-\pi_{n}\right) K^{\prime}(\varphi)+K^{\prime}(\varphi)\left(I-\pi_{n}\right) K^{\prime}\left(\pi_{n} \varphi\right) \pi_{n}
\end{aligned}
$$

If $\pi_{n}$ is the orthogonal projection, then by [13, Theorem 4.1], we obtain

$$
\left\|K^{\prime}(\varphi)\left(I-\pi_{n}\right) K^{\prime}(\varphi)\right\|=O\left(h^{2 \beta}\right) .
$$

In the case of the interpolatory projection, the above estimate is obtained by appealing to [13, Theorem 4.2]. On the other hand,

$$
\begin{aligned}
& K^{\prime}(\varphi)\left(I-\pi_{n}\right) K^{\prime}\left(\pi_{n} \varphi\right) \pi_{n} \\
& =K^{\prime}(\varphi)\left(I-\pi_{n}\right)\left(K^{\prime}\left(\pi_{n} \varphi\right)-K^{\prime}(\varphi)\right) \pi_{n}+K^{\prime}(\varphi)\left(I-\pi_{n}\right) K^{\prime}(\varphi) \pi_{n} .
\end{aligned}
$$

Since, by (2.1),

$$
\left\|K^{\prime}\left(\pi_{n} \varphi\right)-K^{\prime}(\varphi)\right\| \leq \gamma\left\|\pi_{n} \varphi-\varphi\right\|_{\infty} \leq \gamma C_{1}\left\|\varphi^{(\beta)}\right\|_{\infty} h^{\beta}
$$

and $\left\|\pi_{n}\right\|$ are uniformly bounded, it follows that

$$
\left\|K^{\prime}(\varphi)\left(I-\pi_{n}\right)\left(K^{\prime}\left(\pi_{n} \varphi\right)-K^{\prime}(\varphi)\right) \pi_{n}\right\|=O\left(h^{\beta}\right) .
$$

Using (3.8) and (3.9) we obtain

$$
\left\|K^{\prime}(\varphi)\left(\left(K_{n}^{M}\right)^{\prime}(\varphi)-K^{\prime}(\varphi)\right)\right\|=O\left(h^{\beta}\right) .
$$

By Theorem 2.3 in the case of the orthogonal projection and by Theorem 2.6 in the case of the interpolatory projection at Gauss points, we have

$$
\left\|\varphi_{n}^{M}-\varphi\right\|_{\infty}=O\left(h^{3 \beta}\right)
$$


Hence,

$$
\begin{aligned}
\| K^{\prime}(\varphi)\left(\left(K_{n}^{M}\right)^{\prime}(\varphi)-\right. & \left.K^{\prime}(\varphi)\right)\left(\varphi-\varphi_{n}^{M}\right) \|_{\infty} \\
& \leq\left\|K^{\prime}(\varphi)\left(\left(K_{n}^{M}\right)^{\prime}(\varphi)-K^{\prime}(\varphi)\right)\right\|\left\|\varphi-\varphi_{n}^{M}\right\|_{\infty} \\
& =O\left(h^{4 \beta}\right),
\end{aligned}
$$

which completes the proof.

Theorem 3.5. Let $\pi_{n}$ be either the orthogonal projection or the interpolatory projection at $r+1$ Gauss points. Let $\alpha \geq 1$. In the case of the orthogonal projection, assume that $\kappa \in C^{\alpha}(\Omega)$,

$$
\frac{\partial \kappa}{\partial u} \in C^{2 \alpha}(\Omega)
$$

and $f \in C^{\alpha}[0,1]$. In the case of the interpolatory projection at Gauss points, assume that

$$
\kappa, \frac{\partial \kappa}{\partial u} \in C^{2 \alpha}(\Omega)
$$

and $f \in C^{2 \alpha}[0,1]$. Let $\varphi$ be the unique solution of (1.1) and $\varphi_{n}^{M}$ the unique solution of $(1.2)$ in $B\left(\varphi, \delta_{0}\right)$. Assume that 1 is not an eigenvalue of $K^{\prime}(\varphi)$. Then

$$
\left\|\widetilde{\varphi}_{n}^{M}-\varphi\right\|_{\infty}=O\left(h^{4 \beta}\right)
$$

Proof. From (1.1) and (3.1), we obtain

$$
\widetilde{\varphi}_{n}^{M}-\varphi=K\left(\varphi_{n}^{M}\right)-K(\varphi) .
$$

Recall from (2.4) that, for $n$ large enough,

$$
K\left(\varphi_{n}^{M}\right)-K(\varphi)=K^{\prime}(\varphi)\left(\varphi_{n}^{M}-\varphi\right)+R\left(\varphi_{n}^{M}-\varphi\right)
$$

with

$$
\begin{aligned}
& \left(R\left(\varphi_{n}^{M}-\varphi\right)\right)(s) \\
& \quad=\int_{0}^{1}(1-\theta)\left(K^{\prime \prime}\left(\varphi+\theta\left(\varphi_{n}^{M}-\varphi\right)\right)\left(\varphi_{n}^{M}-\varphi\right)^{2}\right)(s) d \theta, s \in[0,1] .
\end{aligned}
$$


Then

$$
\left\|R\left(\varphi_{n}^{M}-\varphi\right)\right\|_{\infty} \leq \frac{C_{9}}{2}\left(\left\|\varphi_{n}^{M}-\varphi\right\|_{\infty}\right)^{2} .
$$

Since by Theorem 2.3 in the case of the orthogonal projection and by Theorem 2.6 in the case of the interpolatory projection at the Gauss points,

$$
\left\|\varphi_{n}^{M}-\varphi\right\|_{\infty}=O\left(h^{3 \beta}\right)
$$

it follows that

$$
\left\|R\left(\varphi_{n}^{M}-\varphi\right)\right\|_{\infty}=O\left(h^{6 \beta}\right) .
$$

Define

$$
B_{n}(x)=\varphi-\left(I-K^{\prime}(\varphi)\right)^{-1}\left[K(\varphi)-K^{\prime}(\varphi) \varphi-K_{n}^{M}(x)+K^{\prime}(\varphi) x\right] .
$$

Then

$$
\begin{aligned}
B_{n}(x) & =x \\
& \Longleftrightarrow\left(I-K^{\prime}(\varphi)\right) \varphi-\left[K(\varphi)-K^{\prime}(\varphi) \varphi-K_{n}^{M}(x)+K^{\prime}(\varphi) x\right] \\
& =\left(I-K^{\prime}(\varphi)\right) x \\
& \Longleftrightarrow x-K_{n}^{M}(x)=\varphi-K(\varphi)=f .
\end{aligned}
$$

Thus, $\varphi_{n}^{M}$ is a fixed point of $B_{n}$, that is,

$$
B_{n}\left(\varphi_{n}^{M}\right)=\varphi_{n}^{M} .
$$

Note that

$$
\begin{aligned}
\varphi_{n}^{M}-\varphi & =B_{n}\left(\varphi_{n}^{M}\right)-\varphi \\
& =-\left(I-K^{\prime}(\varphi)\right)^{-1}\left[K(\varphi)-K^{\prime}(\varphi) \varphi-K_{n}^{M}\left(\varphi_{n}^{M}\right)+K^{\prime}(\varphi) \varphi_{n}^{M}\right] .
\end{aligned}
$$

Since $K^{\prime}(\varphi)$ and $\left(I-K^{\prime}(\varphi)\right)^{-1}$ commute, we obtain

$$
\begin{aligned}
& K^{\prime}(\varphi)\left(\varphi_{n}^{M}-\varphi\right) \\
= & -\left(I-K^{\prime}(\varphi)\right)^{-1} K^{\prime}(\varphi)\left[K(\varphi)-K^{\prime}(\varphi) \varphi-K_{n}^{M}\left(\varphi_{n}^{M}\right)+K^{\prime}(\varphi) \varphi_{n}^{M}\right] .
\end{aligned}
$$

We write

$$
\begin{aligned}
K^{\prime}(\varphi)\left(\varphi_{n}^{M}-\varphi\right)= & -\left(I-K^{\prime}(\varphi)\right)^{-1} K^{\prime}(\varphi)\left[K(\varphi)-K_{n}^{M}(\varphi)\right] \\
& +\left(I-K^{\prime}(\varphi)\right)^{-1} K^{\prime}(\varphi)\left[K_{n}^{M}\left(\varphi_{n}^{M}\right)-K_{n}^{M}(\varphi)\right. \\
& \left.-\left(K_{n}^{M}\right)^{\prime}(\varphi)\left(\varphi_{n}^{M}-\varphi\right)\right] \\
& +\left(I-K^{\prime}(\varphi)\right)^{-1} K^{\prime}(\varphi)\left[\left(\left(K_{n}^{M}\right)^{\prime}(\varphi)-K^{\prime}(\varphi)\right)\left(\varphi_{n}^{M}-\varphi\right)\right] .
\end{aligned}
$$


By Lemma 3.2 the first term in the above expression is of the order of $h^{4 \beta}$. By Lemma 3.3 the second term in the above expression is of the order of $h^{6 \beta}$. Lastly, by Lemma 3.4, the third term in the above expression is of the order of $h^{4 \beta}$. Thus

$$
\left\|K^{\prime}(\varphi)\left(\varphi_{n}^{M}-\varphi\right)\right\|_{\infty}=O\left(h^{4 \beta}\right) .
$$

Combining (3.11), (3.12) and the above estimate, we obtain

$$
\left\|\widetilde{\varphi}_{n}^{M}-\varphi\right\|_{\infty}=\left\|K(\varphi)-K\left(\varphi_{n}^{M}\right)\right\|_{\infty}=O\left(h^{4 \beta}\right),
$$

which completes the proof.

Remark 3.6. In the case when the kernel of $K$ is sufficiently smooth, that is, $\alpha \geq r+1$, we have

$$
\beta=\min \{\alpha, r+1\}=r+1,
$$

and hence

$$
\left\|\varphi-\widetilde{\varphi}_{n}^{M}\right\|_{\infty}=O\left(h^{4 r+4}\right) .
$$

4. Implementation details. Let

$$
\operatorname{dim}\left(X_{n}\right)=N(n)=N .
$$

Let $\left\{e_{n, 1}, \ldots, e_{n, N}\right\}$ be an ordered basis of $X_{n}$ and $\left\{e_{n, 1}^{*}, \ldots, e_{n, N}^{*}\right\}$ the adjoint basis, that is, for $i, j=1, \ldots, N$,

$$
\left\langle e_{n, i}, e_{n, j}^{*}\right\rangle= \begin{cases}1 & \text { if } i=j, \\ 0 & \text { if } i \neq j .\end{cases}
$$

Let $X=L^{2}[0,1]$ or $X=C[0,1]$ and $\pi_{n}: X \rightarrow X_{n}$ be the projection defined by

$$
\pi_{n} x=\sum_{j=1}^{N}\left\langle x, e_{n, j}^{*}\right\rangle e_{n, j} .
$$


Let $\varphi_{n}^{M}$ be the solution of (1.2), and define

$$
\psi_{n}=\pi_{n} \varphi_{n}^{M} .
$$

Recall from (1.2) and (1.3) that

$$
\varphi_{n}^{M}-\pi_{n} K\left(\varphi_{n}^{M}\right)-K\left(\pi_{n} \varphi_{n}^{M}\right)+\pi_{n} K\left(\pi_{n} \varphi_{n}^{M}\right)=f .
$$

Hence,

$$
\left(I-\pi_{n}\right) \varphi_{n}^{M}=\left(I-\pi_{n}\right)\left(K\left(\psi_{n}\right)+f\right)
$$

and

$$
\psi_{n}-\pi_{n} K\left(\varphi_{n}^{M}\right)=\pi_{n} f
$$

that is,

$$
\psi_{n}-\pi_{n} K\left(\psi_{n}+\left(I-\pi_{n}\right)\left(K\left(\psi_{n}\right)+f\right)\right)=\pi_{n} f
$$

Define

$$
F_{n}(y)=y-\pi_{n} K\left(y+\left(I-\pi_{n}\right)(K(y)+f)\right)-\pi_{n} f, \quad y \in X_{n} .
$$

The Fréchet derivative of $F_{n}$ is given by

$$
\left(F_{n}\right)^{\prime}(y) h=h-\pi_{n} K^{\prime}\left(y+\left(I-\pi_{n}\right)(K(y)+f)\right)\left(I+\left(I-\pi_{n}\right) K^{\prime}(y)\right) h .
$$

Equation (4.1) is equivalent to

$$
F_{n}\left(\psi_{n}\right)=0,
$$

and it is iteratively solved by applying the Newton-Kantorovich method. Let $\psi_{n}^{(0)}$ be an initial approximation. The iterates $\psi_{n}^{(k)}, k=1,2, \ldots$, are defined as follows.

$$
\begin{aligned}
\psi_{n}^{(k+1)}-\pi_{n} K^{\prime}\left(\varphi_{n}^{(k)}\right) \psi_{n}^{(k+1)}- & \pi_{n} K^{\prime}\left(\varphi_{n}^{(k)}\right)\left(I-\pi_{n}\right) K^{\prime}\left(\psi_{n}^{(k)}\right) \psi_{n}^{(k+1)} \\
= & \pi_{n}\left(K\left(\varphi_{n}^{(k)}\right)+f\right)-\pi_{n} K^{\prime}\left(\varphi_{n}^{(k)}\right) \psi_{n}^{(k)} \\
& -\pi_{n} K^{\prime}\left(\varphi_{n}^{(k)}\right)\left(I-\pi_{n}\right) K^{\prime}\left(\psi_{n}^{(k)}\right) \psi_{n}^{(k)},
\end{aligned}
$$

where

$$
\varphi_{n}^{(k)}=\psi_{n}^{(k)}+\left(I-\pi_{n}\right)\left(K\left(\psi_{n}^{(k)}\right)+f\right) .
$$


Since $\psi_{n}^{(k)} \in X_{n}$, we can write

$$
\psi_{n}^{(k)}=\sum_{j=1}^{N}\left\langle\psi_{n}^{(k)}, e_{n, j}^{*}\right\rangle e_{n, j}=\sum_{j=1}^{N} x_{n}^{(k)}(j) e_{n, j} .
$$

The system of equations (4.2) is then equivalent to the following system of linear equations of size $N$ :

$$
\left(I-A_{n}^{(k)}-B_{n}^{(k)}\right) x_{n}^{(k+1)}=d_{n}^{(k)},
$$

with

$$
\begin{aligned}
A_{n}^{(k)}(i, j) & =\left\langle K^{\prime}\left(\varphi_{n}^{(k)}\right) e_{n, j}, e_{n, i}^{*}\right\rangle, \\
B_{n}^{(k)}(i, j) & =\left\langle K^{\prime}\left(\varphi_{n}^{(k)}\right)\left(I-\pi_{n}\right) K^{\prime}\left(\psi_{n}^{(k)}\right) e_{n, j}, e_{n, i}^{*}\right\rangle, \\
d_{n}^{(k)}(i) & =\left\langle K\left(\varphi_{n}^{(k)}\right)+f, e_{n, i}^{*}\right\rangle-\left(A_{n}^{(k)} x_{n}^{(k)}\right)(i)-\left(B_{n}^{(k)} x_{n}^{(k)}\right)(i), \\
& i, j=1, \ldots, N .
\end{aligned}
$$

We now consider the system of equations obtained in the collocation/Galerkin method. Recall that

$$
\varphi_{n}^{G}-\pi_{n} K\left(\varphi_{n}^{G}\right)=\pi_{n} f
$$

and hence $\varphi_{n}^{G} \in X_{n}$. Define

$$
G_{n}(y)=y-\pi_{n} K(y)-\pi_{n} f, \quad y \in X_{n},
$$

and solve

$$
G_{n}\left(\varphi_{n}^{G}\right)=0
$$

iteratively by using the Newton-Kantorovich method. Let $\zeta_{n}^{(0)}$ be an initial approximation and the iterates $\zeta_{n}^{(k)}, k=1,2, \ldots$, are as given below.

$$
\zeta_{n}^{(k+1)}-\pi_{n} K^{\prime}\left(\zeta_{n}^{(k)}\right) \zeta_{n}^{(k+1)}=\pi_{n}\left(K\left(\zeta_{n}^{(k)}\right)+f\right)-\pi_{n} K^{\prime}\left(\zeta_{n}^{(k)}\right) \zeta_{n}^{(k)} .
$$

Let

$$
\zeta_{n}^{(k)}=\sum_{j=1}^{N}\left\langle\zeta_{n}^{(k)}, e_{n, j}^{*}\right\rangle e_{n, j}=\sum_{j=1}^{N} y_{n}^{(k)}(j) e_{n, j} .
$$


Then (4.5) is equivalent to the following system of linear equations of size $N$.

$$
\left(I-C_{n}^{(k)}\right) y_{n}^{(k+1)}=r_{n}^{(k)},
$$

with

$$
\begin{gathered}
C_{n}^{(k)}(i, j)=\left\langle K^{\prime}\left(\zeta_{n}^{(k)}\right) e_{n, j}, e_{n, i}^{*}\right\rangle \\
r_{n}^{(k)}(i)=\left\langle K\left(\zeta_{n}^{(k)}\right)+f, e_{n, i}^{*}\right\rangle-\left(C_{n}^{(k)} y_{n}^{(k)}\right)(i), \\
i, j=1, \ldots, N .
\end{gathered}
$$

Remark 4.1. A comparison of (4.4) and (4.6) shows that the latter system is much simpler. Indeed, in the former it is necessary to construct an additional matrix and the right hand side has an extra term. From equation (4.3), it is seen that the computation of the modified projection solution involves an iteration.

If the kernel is smooth enough and if $\pi_{n}$ is either the orthogonal projection or the interpolatory projection at Gauss points, then by (2.18),

$$
\left\|\varphi_{n}^{M}-\varphi\right\|_{\infty}=O\left(h^{3 r+3}\right)
$$

and by (3.13),

$$
\left\|\widetilde{\varphi}_{n}^{M}-\varphi\right\|_{\infty}=O\left(h^{4 r+4}\right) .
$$

Recall from (1.4) that

$$
\left\|\varphi_{n}^{G}-\varphi\right\|_{\infty}=O\left(h^{r+1}\right), \quad\left\|\varphi_{n}^{C}-\varphi\right\|_{\infty}=O\left(h^{r+1}\right)
$$

and by (1.5),

$$
\left\|\varphi_{n}^{S}-\varphi\right\|_{\infty}=O\left(h^{2 r+2}\right) .
$$

Thus, while the size of the system of equations to be solved remains the same, the order of convergence $2 r+2$ in the iterated collocation/Galerkin solution is improved to $3 r+3$ in the modified projection method, and one step of iteration further improves it to $4 r+4$.

5. Numerical results. We illustrate the convergence results that were obtained in Theorem 2.4, Theorem 2.6 and Theorem 3.5 by two numerical examples. 
We consider $X_{n}$ to be the space of piecewise constant or piecewise linear functions with respect to the following uniform partition of $[0,1]$ :

$$
0<\frac{1}{n}<\frac{2}{n}<\cdots<\frac{n}{n}=1 .
$$

The projection $\pi_{n}$ is chosen to be either an interpolatory projection or the orthogonal projection. In the case of the interpolatory projection, the collocation points are chosen to be the $r+1$ Gauss points in each subinterval of the above partition with $r=0$ (midpoints) or $r=1$. In both the examples, since $\alpha=\infty$, it follows that $\beta=r+1$. Hence, by Theorems 2.6 and 3.5,

$$
\left\|\varphi-\varphi_{n}^{M}\right\|_{\infty}=O\left(h^{3 r+3}\right), \quad\left\|\varphi-\widetilde{\varphi}_{n}^{M}\right\|_{\infty}=O\left(h^{4 r+4}\right) .
$$

Also, from [4, equation (7.3)],

$$
\left\|\varphi-\varphi_{n}^{C}\right\|_{\infty}=O\left(h^{r+1}\right), \quad\left\|\varphi-\varphi_{n}^{S}\right\|_{\infty}=O\left(h^{2 r+2}\right) .
$$

5.1. Example 1. Consider the following Hammerstein integral operator with a degenerate kernel:

$$
K(x)(s)=\int_{0}^{1} p(s) q(t) x^{2}(t) d t, \quad s \in[0,1],
$$

where

$$
p(t)=\cos (11 \pi t), \quad q(t)=\sin (11 \pi t) .
$$

Then $K: L^{\infty}[0,1] \rightarrow C[0,1]$ is compact and

$$
\varphi-K(\varphi)=f
$$

has a unique solution for $f \in C[0,1]$. We choose

$$
f(s)=\left(1-\frac{2}{33 \pi}\right) \cos (11 \pi s), \quad s \in[0,1]
$$

so that

$$
\varphi(s)=\cos (11 \pi s), \quad s \in[0,1] .
$$


The Fréchet derivative of $K$ is given by

$$
K^{\prime}(x) h(s)=2 p(s) \int_{0}^{1} q(t) x(t) h(t) d t, \quad h \in L^{\infty}[0,1], s \in[0,1]
$$

For this very special example, it was possible to perform various integrations exactly.

5.1.1. Collocation at Gauss points. For $r=0,1$, let $X_{n}$ be the space of piecewise polynomials of degree $\leq r$ with respect to the partition (5.1). The collocation points are chosen to be $r+1$ Gauss points in each subinterval. The expected orders of convergence are as follows:

$$
\delta_{C}=r+1, \quad \delta_{S}=2 r+2, \quad \delta_{M}=3 r+3, \quad \delta_{M I}=4 r+4
$$

The results are given in Tables 5.1-5.6.

TABLE 5.1. $s=1 / 3 ; r=0$.

\begin{tabular}{|c|cc|cc|cc|cc|}
\hline$n$ & $\left|\varphi(s)-\varphi_{n}^{C}(s)\right|$ & $\delta_{C}$ & $\left|\varphi(s)-\varphi_{n}^{S}(s)\right|$ & $\delta_{S}$ & $\left|\varphi(s)-\varphi_{n}^{M}(s)\right|$ & $\delta_{M}$ & $\left|\varphi(s)-\widetilde{\varphi}_{n}^{M}(s)\right|$ & $\delta_{M I}$ \\
\hline 40 & $1.20 \times 10^{-1}$ & & $7.67 \times 10^{-4}$ & & $1.74 \times 10^{-4}$ & & $6.69 \times 10^{-7}$ & \\
80 & $6.34 \times 10^{-2}$ & 0.91 & $1.63 \times 10^{-4}$ & 2.22 & $2.00 \times 10^{-5}$ & 3.12 & $4.61 \times 10^{-8}$ & 3.86 \\
160 & $3.09 \times 10^{-2}$ & 1.04 & $3.95 \times 10^{-5}$ & 2.05 & $2.34 \times 10^{-6}$ & 3.10 & $2.91 \times 10^{-9}$ & 3.98 \\
320 & $1.57 \times 10^{-2}$ & 0.98 & $9.78 \times 10^{-6}$ & 2.01 & $2.95 \times 10^{-7}$ & 2.99 & $1.83 \times 10^{-10}$ & 4.00 \\
640 & $7.78 \times 10^{-2}$ & 1.01 & $2.44 \times 10^{-6}$ & 2.00 & $3.64 \times 10^{-7}$ & 3.01 & $1.14 \times 10^{-11}$ & 4.00 \\
\hline
\end{tabular}

TABLE 5.2. $s=1 / 3 ; r=1$.

\begin{tabular}{|c|cc|cc|cc|cc|}
\hline$n$ & $\left|\varphi(s)-\varphi_{n}^{C}(s)\right|$ & $\delta_{C}$ & $\left|\varphi(s)-\varphi_{n}^{S}(s)\right|$ & $\delta_{S}$ & $\left|\varphi(s)-\varphi_{n}^{M}(s)\right|$ & $\delta_{M}$ & $\left|\varphi(s)-\widetilde{\varphi}_{n}^{M}(s)\right|$ & $\delta_{M I}$ \\
\hline 40 & $1.20 \times 10^{-2}$ & & $5.14 \times 10^{-5}$ & & $1.19 \times 10^{-6}$ & & $5.83 \times 10^{-9}$ & \\
80 & $2.37 \times 10^{-3}$ & 2.34 & $2.98 \times 10^{-6}$ & 4.11 & $1.36 \times 10^{-8}$ & 6.45 & $1.98 \times 10^{-11}$ & 8.20 \\
160 & $6.75 \times 10^{-4}$ & 1.81 & $1.83 \times 10^{-7}$ & 4.02 & $2.37 \times 10^{-10}$ & 5.84 & $7.45 \times 10^{-14}$ & 8.04 \\
320 & $1.59 \times 10^{-4}$ & 2.09 & $1.14 \times 10^{-8}$ & 4.01 & $3.47 \times 10^{-12}$ & 6.09 & $2.78 \times 10^{-16}$ & 8.08 \\
640 & $4.09 \times 10^{-5}$ & 1.95 & $7.11 \times 10^{-9}$ & 4.00 & $5.57 \times 10^{-14}$ & 6.00 & 0 & - \\
\hline
\end{tabular}


TABLE 5.3. $s=0 ; r=0$.

\begin{tabular}{|c|cc|cc|cc|cc|}
\hline$n$ & $\left|\varphi(s)-\varphi_{n}^{C}(s)\right|$ & $\delta_{C}$ & $\left|\varphi(s)-\varphi_{n}^{S}(s)\right|$ & $\delta_{S}$ & $\left|\varphi(s)-\varphi_{n}^{M}(s)\right|$ & $\delta_{M}$ & $\left|\varphi(s)-\widetilde{\varphi}_{n}^{M}(s)\right|$ & $\delta_{M I}$ \\
\hline 40 & $9.05 \times 10^{-2}$ & & $1.53 \times 10^{-3}$ & & $1.36 \times 10^{-4}$ & & $1.34 \times 10^{-6}$ & \\
80 & $2.29 \times 10^{-2}$ & 1.98 & $3.27 \times 10^{-4}$ & 2.23 & $7.40 \times 10^{-6}$ & 4.20 & $9.23 \times 10^{-8}$ & 3.86 \\
160 & $5.75 \times 10^{-3}$ & 2.00 & $7.89 \times 10^{-5}$ & 2.05 & $4.48 \times 10^{-7}$ & 4.06 & $5.83 \times 10^{-9}$ & 3.98 \\
320 & $1.44 \times 10^{-3}$ & 2.00 & $1.96 \times 10^{-5}$ & 2.01 & $2.78 \times 10^{-8}$ & 4.01 & $3.65 \times 10^{-10}$ & 4.00 \\
640 & $3.60 \times 10^{-4}$ & 2.00 & $4.88 \times 10^{-6}$ & 2.00 & $1.73 \times 10^{-9}$ & 4.00 & $2.28 \times 10^{-11}$ & 4.00 \\
\hline
\end{tabular}

TABLE 5.4. $s=0 ; r=1$.

\begin{tabular}{|c|cc|cc|cc|cc|}
\hline$n$ & $\left|\varphi(s)-\varphi_{n}^{C}(s)\right|$ & $\delta_{C}$ & $\left|\varphi(s)-\varphi_{n}^{S}(s)\right|$ & $\delta_{S}$ & $\left|\varphi(s)-\varphi_{n}^{M}(s)\right|$ & $\delta_{M}$ & $\left|\varphi(s)-\widetilde{\varphi}_{n}^{M}(s)\right|$ & $\delta_{M I}$ \\
\hline 40 & $5.89 \times 10^{-2}$ & & $1.03 \times 10^{-4}$ & & $1.65 \times 10^{-6}$ & & $1.17 \times 10^{-8}$ & \\
80 & $1.53 \times 10^{-2}$ & 1.94 & $5.95 \times 10^{-6}$ & 4.11 & $2.39 \times 10^{-8}$ & 6.11 & $3.96 \times 10^{-10}$ & 8.20 \\
160 & $3.87 \times 10^{-3}$ & 1.99 & $3.66 \times 10^{-7}$ & 4.02 & $3.66 \times 10^{-10}$ & 6.02 & $1.45 \times 10^{-13}$ & 8.04 \\
320 & $9.71 \times 10^{-4}$ & 2.00 & $2.28 \times 10^{-8}$ & 4.01 & $5.70 \times 10^{-12}$ & 6.01 & $5.55 \times 10^{-16}$ & 8.08 \\
640 & $2.43 \times 10^{-4}$ & 2.00 & $1.42 \times 10^{-9}$ & 4.00 & $8.77 \times 10^{-14}$ & 6.02 & 0 & - \\
\hline
\end{tabular}

TABLE 5.5. $s=0.4 ; r=0$.

\begin{tabular}{|c|cc|cc|cc|cc|}
\hline$n$ & $\left|\varphi(s)-\varphi_{n}^{C}(s)\right|$ & $\delta_{C}$ & $\left|\varphi(s)-\varphi_{n}^{S}(s)\right|$ & $\delta_{S}$ & $\left|\varphi(s)-\varphi_{n}^{M}(s)\right|$ & $\delta_{M}$ & $\left|\varphi(s)-\widetilde{\varphi}_{n}^{M}(s)\right|$ & $\delta_{M I}$ \\
\hline 40 & $2.80 \times 10^{-2}$ & & $4.74 \times 10^{-4}$ & & $4.21 \times 10^{-5}$ & & $4.14 \times 10^{-7}$ & \\
80 & $7.08 \times 10^{-3}$ & 1.98 & $1.01 \times 10^{-4}$ & 2.23 & $2.29 \times 10^{-6}$ & 4.20 & $2.85 \times 10^{-8}$ & 3.86 \\
160 & $1.78 \times 10^{-3}$ & 2.00 & $2.44 \times 10^{-5}$ & 2.05 & $1.38 \times 10^{-7}$ & 4.04 & $1.80 \times 10^{-9}$ & 3.98 \\
320 & $4.44 \times 10^{-4}$ & 2.00 & $6.04 \times 10^{-6}$ & 2.01 & $8.58 \times 10^{-9}$ & 4.01 & $1.12 \times 10^{-10}$ & 4.00 \\
640 & $1.11 \times 10^{-4}$ & 2.00 & $1.51 \times 10^{-6}$ & 2.00 & $5.35 \times 10^{-10}$ & 4.00 & $7.06 \times 10^{-12}$ & 4.00 \\
\hline
\end{tabular}

TABLE 5.6. $s=0.4 ; r=1$.

\begin{tabular}{|c|cc|cc|cc|cc|}
\hline$n$ & $\left|\varphi(s)-\varphi_{n}^{C}(s)\right|$ & $\delta_{C}$ & $\left|\varphi(s)-\varphi_{n}^{S}(s)\right|$ & $\delta_{S}$ & $\left|\varphi(s)-\varphi_{n}^{M}(s)\right|$ & $\delta_{M}$ & $\left|\varphi(s)-\widetilde{\varphi}_{n}^{M}(s)\right|$ & $\delta_{M I}$ \\
\hline 40 & $1.82 \times 10^{-2}$ & & $3.17 \times 10^{-5}$ & & $3.44 \times 10^{-6}$ & & $3.60 \times 10^{-9}$ & \\
80 & $4.74 \times 10^{-3}$ & 1.94 & $1.84 \times 10^{-6}$ & 4.11 & $3.92 \times 10^{-8}$ & 6.45 & $1.22 \times 10^{-11}$ & 8.20 \\
160 & $1.19 \times 10^{-3}$ & 1.99 & $1.13 \times 10^{-7}$ & 4.02 & $5.15 \times 10^{-10}$ & 6.25 & $4.63 \times 10^{-14}$ & 8.04 \\
320 & $3.00 \times 10^{-4}$ & 2.00 & $7.04 \times 10^{-9}$ & 4.01 & $7.30 \times 10^{-12}$ & 6.14 & $1.66 \times 10^{-16}$ & 8.12 \\
640 & $7.51 \times 10^{-5}$ & 2.00 & $4.39 \times 10^{-10}$ & 4.00 & $1.08 \times 10^{-13}$ & 6.00 & 0 & - \\
\hline
\end{tabular}

Remark 5.1. It can be seen that, for $s=1 / 3$, the computed orders of convergence match well with the theoretically predicted values. In the case of $r=0$, or the partition points $s=0$ and $s=0.4$, the computed orders of convergence $\delta_{C}$, in the collocation method and $\delta_{M}$, in the modified projection method are respectively 2 and 4 . These values are 
better than the predicted values. Note that, even though the orders of convergence in the collocation and the iterated collocation method are the same, the error in the iterated collocation method is smaller than the collocation error. The same observation is valid for the modified projection and the iterated modified projection methods.

It can be seen from Table 5.1 that the approximation in the iterated modified projection method with $n=40$ is better than the iterated collocation approximation with $n=640$.

5.1.2. Collocation at partition points. Let $X_{n}$ be the space of continuous piecewise linear functions $(r=1)$ with respect to the partition (5.1). Then the dimension of $X_{n}$ is equal to $n+1$. The collocation points in this case are chosen to be the partition points

$$
t_{i}=\frac{i-1}{n}, \quad i=1, \ldots, n+1
$$

The expected orders of convergence are as follows:

$$
\delta_{C}=\delta_{S}=2, \quad \delta_{M}=\delta_{M I}=4
$$

The results are given in Tables 5.7-5.9.

Remark 5.2. It is seen from the results in Tables 5.7-5.9 that the computed orders of convergence match well with the expected values.

For $s=1 / 3$, note that the error in the iterated modified projection is smaller as compared to the modified projection method, even though the orders of convergence are the same. The same phenomenon occurs for the collocation and the iterated collocation methods.

For $s=0$ and $s=0.4$, the values in the second and the fourth columns of Tables 5.8 and 5.9 are identical. Similarly, the values in the sixth and eighth columns are identical. This is expected, since $s=0$ and $s=0.4$ are the collocation points and

$$
\pi_{n} \varphi_{n}^{S}=\varphi_{n}^{C}, \quad \pi_{n} \widetilde{\varphi}_{n}^{M}=\pi_{n} \varphi_{n}^{M}
$$

As in the case of piecewise constant functions, the approximation $\widetilde{\varphi}_{n}^{M}$ in the iterated modified projection seems to be the best. 
TABLE 5.7. $\mathrm{s}=1 / 3$.

\begin{tabular}{|c|cc|cc|cc|cc|}
\hline$n$ & $\left|\varphi(s)-\varphi_{n}^{C}(s)\right|$ & $\delta_{C}$ & $\left|\varphi(s)-\varphi_{n}^{S}(s)\right|$ & $\delta_{S}$ & $\left|\varphi(s)-\varphi_{n}^{M}(s)\right|$ & $\delta_{M}$ & $\left|\varphi(s)-\widetilde{\varphi}_{n}^{M}(s)\right|$ & $\delta_{M I}$ \\
\hline 40 & $4.98 \times 10^{-2}$ & & $2.69 \times 10^{-3}$ & & $1.14 \times 10^{-4}$ & & $3.00 \times 10^{-6}$ & \\
80 & $1.02 \times 10^{-2}$ & 2.29 & $7.50 \times 10^{-4}$ & 1.84 & $5.82 \times 10^{-6}$ & 4.30 & $1.87 \times 10^{-7}$ & 4.00 \\
160 & $2.89 \times 10^{-3}$ & 1.82 & $1.93 \times 10^{-4}$ & 1.96 & $4.15 \times 10^{-7}$ & 3.81 & $1.17 \times 10^{-8}$ & 4.00 \\
320 & $6.83 \times 10^{-4}$ & 2.08 & $4.86 \times 10^{-5}$ & 1.99 & $2.45 \times 10^{-8}$ & 4.08 & $7.31 \times 10^{-10}$ & 4.00 \\
640 & $1.76 \times 10^{-4}$ & 1.96 & $1.22 \times 10^{-5}$ & 2.00 & $1.58 \times 10^{-9}$ & 3.96 & $4.57 \times 10^{-11}$ & 4.00 \\
\hline
\end{tabular}

TABLE 5.8. $\mathrm{s}=0$.

\begin{tabular}{|c|cc|cc|cc|cc|}
\hline$n$ & $\left|\varphi(s)-\varphi_{n}^{C}(s)\right|$ & $\delta_{C}$ & $\left|\varphi(s)-\varphi_{n}^{S}(s)\right|$ & $\delta_{S}$ & $\left|\varphi(s)-\varphi_{n}^{M}(s)\right|$ & $\delta_{M}$ & $\left|\varphi(s)-\widetilde{\varphi}_{n}^{M}(s)\right|$ & $\delta_{M I}$ \\
\hline 40 & $5.38 \times 10^{-3}$ & & $5.38 \times 10^{-3}$ & & $5.99 \times 10^{-6}$ & & $5.99 \times 10^{-6}$ & \\
80 & $1.50 \times 10^{-3}$ & 1.84 & $1.50 \times 10^{-3}$ & 1.84 & $3.73 \times 10^{-7}$ & 4.00 & $3.73 \times 10^{-7}$ & 4.00 \\
160 & $3.86 \times 10^{-4}$ & 1.96 & $3.86 \times 10^{-4}$ & 1.96 & $2.34 \times 10^{-8}$ & 4.00 & $2.34 \times 10^{-8}$ & 4.00 \\
320 & $9.73 \times 10^{-5}$ & 1.99 & $9.73 \times 10^{-5}$ & 1.99 & $1.46 \times 10^{-9}$ & 4.00 & $1.46 \times 10^{-9}$ & 4.00 \\
640 & $2.44 \times 10^{-5}$ & 2.00 & $2.44 \times 10^{-5}$ & 2.00 & $9.14 \times 10^{-11}$ & 4.00 & $9.14 \times 10^{-11}$ & 4.00 \\
\hline
\end{tabular}

TABLE 5.9. $\mathrm{s}=0.4$.

\begin{tabular}{|c|cc|cc|cc|cc|}
\hline$n$ & $\left|\varphi(s)-\varphi_{n}^{C}(s)\right|$ & $\delta_{C}$ & $\left|\varphi(s)-\varphi_{n}^{S}(s)\right|$ & $\delta_{S}$ & $\left|\varphi(s)-\varphi_{n}^{M}(s)\right|$ & $\delta_{M}$ & $\left|\varphi(s)-\widetilde{\varphi}_{n}^{M}(s)\right|$ & $\delta_{M I}$ \\
\hline 40 & $1.66 \times 10^{-3}$ & & $1.66 \times 10^{-3}$ & & $1.85 \times 10^{-6}$ & & $1.85 \times 10^{-6}$ & \\
80 & $4.64 \times 10^{-4}$ & 1.84 & $4.64 \times 10^{-4}$ & 1.84 & $1.15 \times 10^{-7}$ & 4.00 & $1.15 \times 10^{-7}$ & 4.00 \\
160 & $1.19 \times 10^{-4}$ & 1.96 & $1.19 \times 10^{-4}$ & 1.96 & $7.22 \times 10^{-9}$ & 4.00 & $7.22 \times 10^{-9}$ & 4.00 \\
320 & $3.01 \times 10^{-5}$ & 1.99 & $3.01 \times 10^{-5}$ & 1.99 & $4.52 \times 10^{-10}$ & 4.00 & $4.52 \times 10^{-10}$ & 4.00 \\
640 & $7.53 \times 10^{-6}$ & 2.00 & $7.53 \times 10^{-6}$ & 2.00 & $2.82 \times 10^{-11}$ & 4.00 & $2.82 \times 10^{-11}$ & 4.00 \\
\hline
\end{tabular}

5.2. Example 2. Consider

$$
\varphi(s)-\int_{0}^{1} \frac{d s}{s+t+\varphi(t)}=f(s), \quad 0 \leq s \leq 1
$$

where $f$ is so chosen that

$$
\varphi(t)=\frac{1}{t+c}, \quad c>0
$$

is a solution of (5.3).

We choose $c=1$ and $c=0.1$. The results are worse in the case of $c=0.1$, since the exact solution is ill behaved. 
In this example, we need to evaluate integrals numerically. If $X_{n}$ is the space of piecewise constant functions with respect to the partition (5.1), then in both the cases of interpolatory projection at the midpoints of the subintervals and of the orthogonal projection, the order of convergence in the iterated version of the modified projection method is $1 / n^{4}$ and it is the least as compared to the other three methods which we consider. Hence, we choose composite Simpson rule with respect to the partition (5.1) to evaluate the integrals numerically. If $X_{n}$ is the space of piecewise linear functions with respect to the partition (5.1), and the interpolation points are Gauss 2 points, then the order of convergence in the iterated version of the modified projection method is $1 / n^{8}$. In this case, we choose composite Gauss 2 point rule with respect to a uniform partition with $n^{2}$ subintervals as the approximate quadrature rule.

5.2.1. Collocation at Gauss points. For $r=0,1$, let $X_{n}$ be the space of piecewise polynomials of degree $\leq r$ with respect to the partition (5.1). The collocation points are chosen to be $r+1$ Gauss points in each subinterval. The expected orders of convergence are as follows:

$$
\delta_{C}=r+1, \quad \delta_{S}=2 r+2, \quad \delta_{M}=3 r+3, \quad \delta_{M I}=4 r+4 .
$$

The results are given in Tables 5.10-5.13.

TABLE 5.10. $\varphi(t)=1 /(t+1) ; r=0$.

\begin{tabular}{|c|cc|cc|cc|cc|}
\hline$n$ & $\left\|\varphi-\varphi_{n}^{C}\right\|_{\infty}$ & $\delta_{C}$ & $\left\|\varphi-\varphi_{n}^{S}\right\|$ & $\delta_{S}$ & $\left\|\varphi-\varphi_{n}^{M}\right\|_{\infty}$ & $\delta_{M}$ & $\left\|\varphi-\widetilde{\varphi}_{n}^{M}\right\|_{\infty}$ & $\delta_{M I}$ \\
\hline 2 & $1.09 \times 10^{-1}$ & & $2.64 \times 10^{-3}$ & & $8.46 \times 10^{-4}$ & & $2.38 \times 10^{-5}$ & \\
4 & $5.67 \times 10^{-2}$ & 0.95 & $6.07 \times 10^{-4}$ & 2.12 & $1.03 \times 10^{-4}$ & 3.04 & $1.37 \times 10^{-6}$ & 4.12 \\
8 & $2.85 \times 10^{-2}$ & 0.99 & $1.44 \times 10^{-4}$ & 2.07 & $1.24 \times 10^{-5}$ & 3.05 & $8.18 \times 10^{-8}$ & 4.07 \\
16 & $1.37 \times 10^{-2}$ & 1.06 & $3.52 \times 10^{-5}$ & 2.04 & $1.45 \times 10^{-6}$ & 3.09 & $4.99 \times 10^{-9}$ & 4.04 \\
32 & $6.10 \times 10^{-3}$ & 1.17 & $8.67 \times 10^{-6}$ & 2.02 & $1.59 \times 10^{-7}$ & 3.19 & $3.08 \times 10^{-10}$ & 4.02 \\
\hline
\end{tabular}

TABLE 5.11. $\varphi(t)=1 /(t+1) ; r=1$.

\begin{tabular}{|c|cc|cc|cc|cc|}
\hline$n$ & $\left\|\varphi-\varphi_{n}^{C}\right\|_{\infty}$ & $\delta_{C}$ & $\left\|\varphi-\varphi_{n}^{S}\right\|$ & $\delta_{S}$ & $\left\|\varphi-\varphi_{n}^{M}\right\|_{\infty}$ & $\delta_{M}$ & $\left\|\varphi-\widetilde{\varphi}_{n}^{M}\right\|_{\infty}$ & $\delta_{M I}$ \\
\hline 2 & $7.60 \times 10^{-2}$ & & $1.36 \times 10^{-3}$ & & $5.06 \times 10^{-4}$ & & $6.47 \times 10^{-5}$ & \\
4 & $2.64 \times 10^{-2}$ & 1.53 & $8.18 \times 10^{-5}$ & 4.05 & $1.07 \times 10^{-5}$ & 5.56 & $2.09 \times 10^{-7}$ & 8.27 \\
8 & $7.92 \times 10^{-3}$ & 1.74 & $4.68 \times 10^{-6}$ & 4.13 & $1.85 \times 10^{-7}$ & 5.86 & $8.45 \times 10^{-10}$ & 7.95 \\
16 & $2.13 \times 10^{-3}$ & 1.90 & $2.84 \times 10^{-7}$ & 4.04 & $3.07 \times 10^{-9}$ & 5.90 & $3.35 \times 10^{-12}$ & 7.98 \\
32 & $5.17 \times 10^{-4}$ & 2.04 & $1.76 \times 10^{-8}$ & 4.01 & $4.74 \times 10^{-11}$ & 6.02 & $1.34 \times 10^{-14}$ & 7.96 \\
\hline
\end{tabular}


TABLE 5.12. $\varphi(t)=1 /(t+0.1) ; r=0$.

\begin{tabular}{|c|cc|cc|cc|cc|}
\hline$n$ & $\left\|\varphi-\varphi_{n}^{C}\right\|_{\infty}$ & $\delta_{C}$ & $\left\|\varphi-\varphi_{n}^{S}\right\|$ & $\delta_{S}$ & $\left\|\varphi-\varphi_{n}^{M}\right\|_{\infty}$ & $\delta_{M}$ & $\left\|\varphi-\widetilde{\varphi}_{n}^{M}\right\|_{\infty}$ & $\delta_{M I}$ \\
\hline 2 & 5.56 & & $1.12 \times 10^{-3}$ & & $3.64 \times 10^{-4}$ & & $7.80 \times 10^{-6}$ & \\
4 & 3.68 & 0.59 & $6.73 \times 10^{-4}$ & 0.73 & $6.29 \times 10^{-5}$ & 2.53 & $4.20 \times 10^{-7}$ & 4.21 \\
8 & 2.22 & 0.73 & $2.33 \times 10^{-4}$ & 1.53 & $8.85 \times 10^{-6}$ & 2.83 & $2.42 \times 10^{-8}$ & 4.12 \\
16 & 1.19 & 0.90 & $6.76 \times 10^{-5}$ & 1.78 & $1.12 \times 10^{-6}$ & 2.99 & $1.45 \times 10^{-9}$ & 4.06 \\
32 & $5.62 \times 10^{-1}$ & 1.08 & $1.82 \times 10^{-5}$ & 1.89 & $1.27 \times 10^{-7}$ & 3.14 & $8.89 \times 10^{-11}$ & 4.03 \\
\hline
\end{tabular}

TABLE 5.13. $\varphi(t)=1 /(t+0.1) ; r=1$.

\begin{tabular}{|c|cc|cc|cc|cc|}
\hline$n$ & $\left\|\varphi-\varphi_{n}^{C}\right\|_{\infty}$ & $\delta_{C}$ & $\left\|\varphi-\varphi_{n}^{S}\right\|$ & $\delta_{S}$ & $\left\|\varphi-\varphi_{n}^{M}\right\|_{\infty}$ & $\delta_{M}$ & $\left\|\varphi-\widetilde{\varphi}_{n}^{M}\right\|_{\infty}$ & $\delta_{M I}$ \\
\hline 2 & 6.02 & & $1.03 \times 10^{-3}$ & & $9.39 \times 10^{-5}$ & & $1.14 \times 10^{-4}$ & \\
4 & 3.95 & 0.61 & $2.19 \times 10^{-3}$ & -1.08 & $1.19 \times 10^{-4}$ & -0.35 & $2.84 \times 10^{-7}$ & 8.65 \\
8 & 2.17 & 0.87 & $4.08 \times 10^{-4}$ & 2.43 & $3.30 \times 10^{-6}$ & 5.18 & $1.10 \times 10^{-9}$ & 8.01 \\
16 & $9.41 \times 10^{-1}$ & 1.20 & $3.85 \times 10^{-5}$ & 3.40 & $4.99 \times 10^{-8}$ & 6.05 & $4.35 \times 10^{-12}$ & 7.99 \\
32 & $3.20 \times 10^{-1}$ & 1.55 & $2.80 \times 10^{-6}$ & 3.78 & $7.00 \times 10^{-10}$ & 6.16 & $1.78 \times 10^{-14}$ & 7.93 \\
\hline
\end{tabular}

5.2.2. Orthogonal projection. Let $X_{n}$ be the space of piecewise constant functions and $\pi_{n}$ the orthogonal projection from $L^{2}[0,1]$ onto $X_{n}$. The expected orders of convergence are as follows:

$$
\delta_{G}=1, \quad \delta_{S}=2, \quad \delta_{M}=3, \quad \delta_{M I}=4
$$

The results are given in Tables 5.14-5.15.

TABLE 5.14. $\varphi(t)=1 /(t+1)$.

\begin{tabular}{|c|cc|cc|cc|cc|}
\hline$n$ & $\left\|\varphi-\varphi_{n}^{G}\right\|_{\infty}$ & $\delta_{G}$ & $\left\|\varphi-\varphi_{n}^{S}\right\|$ & $\delta_{S}$ & $\left\|\varphi-\varphi_{n}^{M}\right\|_{\infty}$ & $\delta_{M}$ & $\left\|\varphi-\widetilde{\varphi}_{n}^{M}\right\|_{\infty}$ & $\delta_{M I}$ \\
\hline 2 & $1.85 \times 10^{-1}$ & & $7.15 \times 10^{-3}$ & & $3.92 \times 10^{-3}$ & & $1.75 \times 10^{-4}$ & \\
4 & $1.05 \times 10^{-1}$ & 0.82 & $1.48 \times 10^{-3}$ & 2.28 & $4.90 \times 10^{-4}$ & 3.00 & $9.34 \times 10^{-6}$ & 4.22 \\
8 & $5.58 \times 10^{-2}$ & 0.91 & $3.17 \times 10^{-4}$ & 2.21 & $5.86 \times 10^{-5}$ & 3.06 & $5.41 \times 10^{-7}$ & 4.11 \\
16 & $2.83 \times 10^{-2}$ & 0.98 & $7.21 \times 10^{-5}$ & 2.14 & $6.90 \times 10^{-6}$ & 3.09 & $3.26 \times 10^{-8}$ & 4.05 \\
32 & $1.36 \times 10^{-2}$ & 1.05 & $1.71 \times 10^{-5}$ & 2.08 & $7.96 \times 10^{-7}$ & 3.12 & $2.00 \times 10^{-9}$ & 4.03 \\
\hline
\end{tabular}

TABLE 5.15. $\varphi(t)=1 /(t+0.1)$.

\begin{tabular}{|c|cc|cc|cc|cc|}
\hline$n$ & $\left\|\varphi-\varphi_{n}^{G}\right\|_{\infty}$ & $\delta_{G}$ & $\left\|\varphi-\varphi_{n}^{S}\right\|$ & $\delta_{S}$ & $\left\|\varphi-\varphi_{n}^{M}\right\|_{\infty}$ & $\delta_{M}$ & $\left\|\varphi-\widetilde{\varphi}_{n}^{M}\right\|_{\infty}$ & $\delta_{M I}$ \\
\hline 2 & 6.18 & & $3.99 \times 10^{-2}$ & & $7.51 \times 10^{-3}$ & & $8.42 \times 10^{-5}$ & \\
4 & 4.74 & 0.38 & $1.09 \times 10^{-2}$ & 1.87 & $1.07 \times 10^{-3}$ & 2.81 & $4.69 \times 10^{-6}$ & 4.16 \\
8 & 3.33 & 0.51 & $2.91 \times 10^{-3}$ & 1.90 & $1.44 \times 10^{-4}$ & 2.90 & $2.91 \times 10^{-7}$ & 4.01 \\
16 & 2.07 & 0.69 & $7.68 \times 10^{-4}$ & 1.92 & $1.85 \times 10^{-5}$ & 2.96 & $1.85 \times 10^{-8}$ & 3.97 \\
32 & 1.14 & 0.86 & $1.99 \times 10^{-4}$ & 1.95 & $2.25 \times 10^{-6}$ & 3.04 & $1.18 \times 10^{-9}$ & 3.98 \\
\hline
\end{tabular}


Remark 5.3. Note that the computed values of orders of convergence in all the cases are as expected.

\section{REFERENCES}

1. K.E. Atkinson, A survey of numerical methods for solving nonlinear integral equations, J. Int. Eqns. Appl. 4 (1992), 15-46.

2. - The numerical solutions of integral equations of the second kind, Cambridge University Press, Cambridge, 1997.

3. K. Atkinson, I. Graham and I. Sloan, Piecewise continuous collocation for integral equations, SIAM J. Num. Anal. 20 (1983), 172-186.

4. K.E. Atkinson and F.A. Potra, Projection and iterated projection methods for nonlinear integral equations, SIAM J. Numer. Anal. 24 (1987), 1352-1373.

5. — The discrete Galerkin method for nonlinear integral equations, J. Int. Eqns. Appl. 1 (1988), 17-54.

6. C. De Boor and B. Swartz, Collocation at Gaussian points, SIAM J. Numer. Anal. 10 (1973), 582-606.

7. L. Grammont, A Galerkin's perturbation type method to approximate a fixed point of a compact operator, Int. J. Pure Appl. Math. 69 (2011), 1-14.

8. L. Grammont and R.P. Kulkarni, A superconvergent projection method for nonlinear compact operator equations, C.R. Acad. Sci. Paris 342 (2006), 215-218.

9. M.A. Krasnoselskii, Topological methods in the theory of nonlinear integral equations, Pergamon Press, London, 1964.

10. M.A. Krasnoselskii, G.M. Vainikko, P.P. Zabreiko, et al., Approximate solution of operator equations, P. Noordhoff, Groningen, 1972.

11. M.A. Krasnoselskii and P.P. Zabreiko, Geometrical methods of nonlinear analysis, Springer-Verlag, Berlin, 1984.

12. R.P. Kulkarni, A superconvergence result for solutions of compact operator equations, Bull. Austr. Math. Soc. 68 (2003), 517-528.

13. R.P. Kulkarni and N. Gnaneshwar, Spectral refinement using a new projection method, ANZIAM J. 46 (2004), 203-224.

14. G.M. Vainikko, Galerkin's perturbation method and general theory of approximate methods for non-linear equations, USSR Comput. Math. Math Phys. 7 (1967), 1-41.

Université de Lyon, Institut Camille Jordan, 23, rue du Dr Paul MichElon, 42023, Saint-Étienne Cedex 2, France

Email address: laurence.grammont@univ-st-etienne.fr

Department of Mathematics, I.I.T. Bombay, Powai, Mumbai 400076, India Email address: rpk@math.iitb.ac.in

Faculdade de Economia da Universidade do Porto and Centro Mathemática Da Universidade do Porto, Portugal

Email address: pjv@fep.up.pt 